Classification

Physics Abstracts

$51.10-05.60$

\title{
La transformée de Wigner libre, évolution dans une collision
}

\author{
F. Laloë (*) \\ Laboratoire de Physique de l'E.N.S., 24 rue Lhomond, F 75005 Paris, France
}

(Reçu le 5 décembre 1988, accepté le 16 février 1989)

\begin{abstract}
Résumé. - Le but général de la série d'articles dont celui-ci est le premier est de décrire la dynamique hors d'équilibre des gaz quantiques dilués en tenant compte de façon précise des corrélations binaires entre particules; ces corrélations sont généralement ignorées dans les approches théoriques du type Boltzmann. Nous proposons pour cela l'utilisation d'une variante de la transformée de Wigner, la transformée de Wigner libre, qui « efface » les corrélations à courte distance créées par le potentiel d'interaction entre particules. Ainsi, chaque fois qu'avant collision la transformée libre était factorisée, une factorisation subsiste rigoureusement pendant toute la collision; elle se prête donc mieux que la transformée habituelle à une hypothèse de « chaos moléculaire » à la Boltzmann. Une fois connue la transformée libre, les corrélations entre particules peuvent être reconstruites et leurs effets physiques calculés. L'étude de l'évolution de la transformée de Wigner libre dans une collision binaire conduit à des résultats qui s'expriment complètement en fonction des caractéristiques de la matrice $T$ « sur la couche de masse » (matrice $S$, déphasages), même lorsque les particules sont en train d'interagir. En sus des termes habituels de collision où apparaissent comme dans l'équation de Boltzmann les sections efficaces de collision, le calcul donne des termes dus aux effets de retard, et aux effets « de réfraction » dans la collision. Ces termes joueront un rôle essentiel dans l'équation cinétique proposée dans l'article suivant, et dans les corrections du viriel aux propriétés d'équilibre et de transport du gaz.
\end{abstract}

Abstract. - This is the first of a series of articles where we develop a theory for the description of
the dynamics of dilute quantum gases, at or out of equilibrium, with an accurate treatment of the
correlation effects introduced by binary collisions (these correlations are not taken into account
when the Boltzmann equation is used). For this purpose, we propose the introduction of a variant
of the Wigner transform, the "free Wigner transform ", which wipes out the effects of the
potential at short distances. If initially factorized, the entering part of the free transform remains
exactly so during all collision, so that it is better adapted to a molecular chaos assumption than the
ordinary transform. Once the free transform is known, the effects of the short distance
correlations can be reconstructed from it. Therefore, the problem is to find an appropriate
equation of evolution which gives its time variations. With this aim in mind, we study in the
present article the evolution of the free Wigner transform in a binary collision. We show that its
evolution equation includes coefficients which can be exactly obtained from the on shell » terms
of the $T$ matrix ( $S$ matrix, phase shifts), even while the particles are interacting. In addition to the
usual terms which involve the scattering cross sections, as usual with the Boltzmann equation, the

(*) Membre du Laboratoire de Spectroscopie Hertzienne de l'E.N.S., laboratoire associé au CNRS $\mathrm{n}^{\circ} 18$. 
calculation provides other terms which correspond to collision retardation effects and « refraction » effects (refraction of the wave function of the test atom by density gradients of the collision partners). These terms will play an important role in the following article, and introduce the second virial corrections to the equilibrium and transport properties of the gas.

\section{Introduction.}

Cet article fait partie d'une série dont le but général est de décrire la dynamique des gaz quantiques dilués en prenant en compte de façon précise les effets des corrélations à deux corps introduites à courte distance par les interactions dans le système. L'équation de Boltzmann est un outil très puissant pour l'étude de la dynamique des gaz, puisqu'elle permet de tenir compte de l'effet des collisions binaires sur leur évolution, mais elle ignore tous les effets de corrélation entre particules, ce qui n'est pas sans conséquences physiques. Par exemple, si l'on calcule la pression à partir de l'équation de Boltzmann (via la méthode de Chapman Enskog et la construction d'équations hydrodynamiques [1]), on constate que l'on trouve seulement la pression d'un gaz parfait, sans la seconde correction du viriel ; pourtant cette dernière est, elle aussi, due à l'effet des collisions binaires, ce qui montre bien que ces collisions ne sont pas totalement prises en compte dans l'approche de Boltzmann. On peut voir là une certaine incohérence, à laquelle nous nous donnons comme objectif de remédier dans le présent travail.

La méthode habituelle pour obtenir les secondes corrections du viriel à la pression, la capacité calorifique, etc. d'un système dilué part d'un développement en densité de la fonction de partition, ce qui en limite l'application aux systèmes à l'équilibre. Puisque ces corrections ne sont pas obtenues si l'on part de l'équation cinétique de Boltzmann, qui est valable hors d'équilibre, le but que nous nous proposons est donc en quelque sorte de réconcilier descriptions dynamique et statique du système. Nous nous limiterons cependant à l'étude des interactions à deux corps et, en conséquence, des seconds coefficients du viriel ; nous travaillerons dans un cadre complètement quantique, afin de rétablir à partir d'une équation cinétique l'expression exacte des seconds coefficients du viriel donnée par Beth et Uhlenbeck $[2,3]$.

Le point de vue de Boltzmann est de considérer les collisions comme des phénomènes ponctuels, dans le temps (pas de durée) et dans l'espace (pas de portée du potentiel). Evidemment, ceci n'empêche pas de mettre les sections efficaces exactes, calculées quantiquement, dans le second membre de l'équation (le terme de collision) - c'est par exemple l'approche de Waldmann [4]. En mécanique quantique comme classique, le point essentiel pour ce qui nous occupe ici est que, dans ce type de description, les particules ne sont jamais « en train d'interagir », restriction qui élimine certains aspects essentiels des processus collisionnels. Clairement, une telle limitation doit être levée pour le but que nous nous sommes fixés. Il s'agit donc :

(1) d'introduire dans la théorie les effets de retard qui se produisent pendant les collisions, et de tenir compte de l'existence d'une portée non nulle du potentiel d'interaction. A ces effets s'en ajouteront d'autres, de nature purement quantique, que nous appellerons « effets de réfraction ». Considérons un atome donné se propageant dans un gaz de densité non uniforme : en présence d'un gradient de densité (perpendiculaire à la vitesse), l'onde de De Broglie qui lui est associée sera réfractée par un effet de lentille analogue à celui qui se produirait pour un photon se propageant dans un gaz de densité variable. De tels effets vont engendrer dans l'équation cinétique des termes du type "potentiel effectif » qui ne sont évidemment pas pris en compte lorsqu'on utilise l'équation de Boltzmann; 
(2) de tenir compte explicitement dans la théorie de la présence des corrélations à courtes distances, et en particulier de ne pas supposer que la fonction de distribution à deux particules, $f_{\mathrm{II}}$, est simplement le produit $f_{\mathrm{I}} \times f_{\mathrm{I}}$ de fonctions de distribution à une particule, $f_{\mathrm{I}}$.

Nous verrons que cela est effectivement possible, tout en utilisant une fonction de distribution $f$ qui ressemble à $f_{\mathrm{I}}$ mais en diffère cependant de façon essentielle : $f$ décrit le comportement statistique d'une particule quelconque du système lorsqu'elle est éloignée de toutes les autres ; cette condition d'éloignement - qui dépend évidemment de la position de toutes les autres particules - fait de $f$ autre chose qu'une distribution ordinaire à une particule. En général d'ailleurs $f$ ne peut être définie, ni en fonction de $f_{\mathrm{I}}$ seule, ni même de $f_{\text {II }}$, mais uniquement de la distribution $f_{N}$ du système de l'ensemble des particules.

Pour fixer les idées, prenons d'abord un système de deux particules seulement. La fonction $f$ décrira alors simplement le comportement asymptotique à grande distance (relative des deux particules) de la fonction $f_{\mathrm{II}}$ : lorsque les particules sont éloignées, hors de la portée du potentiel d'interaction, on peut supposer que la factorisation $f_{\mathrm{II}}=f \times f$ est réalisée ; c'est une hypothèse qui est bien dans l'esprit de celle de Boltzmann, tout en préservant la possibilité que cette factorisation ne se produise pas à courte distance.

Pour un système comprenant un grand nombre de particules, il n'est pas suffisant de s'assurer que la particule 2 soit éloignée de la particule 1 pour que cette dernière ne soit pas en interaction avec une troisième (pour un grand système, la distribution $f_{\text {II }}$ se factorise d'ailleurs simplement en $f_{\mathrm{I}} \times f_{\mathrm{I}}$ à grande distance mutuelle). En conséquence, et comme nous l'avons deja noté plus haut, la fonction $f$ doit maintenant être définie à partir de la fonction de distribution $f_{N}$ à $N$ particules : $f$ donne la dépendance de $f_{N}$ en fonction des variables dynamiques de la particule 1 dans les régions de l'espace des configurations où la particule 1 est suffisamment éloignée de la particule la plus proche. Supposer que la dépendance de $f_{N}$ en fonction des variables de 1 se factorise de cette façon est une hypothèse qui paraît raisonnable et, comme plus haut, bien dans l'esprit de Boltzmann.

En pratique cependant, nous n'aurons pas besoin pour nos calculs de la définition générale de $f$ à partir de $f_{N}$ : nous raisonnerons au cours de tout cet article sur un système de deux particules seulement, ce qui simplifie le formalisme en évitant de multiples traces partielles ; en conséquence, la définition simple de $f$ à partir de $f_{\text {II }}$ sera suffisante au moins pour le présent travail.

C'est donc cette fonction $f$ qui sera la base de notre théorie. C'est sur $f$ que sera faite la «Stossansatz » de Boltzmann, c'est également $f$ qui apparaîtra dans l'équation cinétique du système. C'est enfin à partir de $f$ que seront calculées les modifications dues aux corrélations à courte distance (en gros, pour un système de deux particules, la différence $\left.\delta f_{\mathrm{II}}=f_{\mathrm{II}}-f \times f\right)$ et les corrections du viriel correspondantes.

Se pose alors bien sûr la question de la définition précise de $f$ à partir de l'opérateur densité $\mathrm{du}$ système, pour toute configuration qui lui est accessible : il ne peut évidemment être question de se contenter d'une définition valable uniquement lorsque les particules sont éloignées si l'on désire rendre compte de l'effet des collisions par une équation cinétique. Nous définirons donc $f$ par extrapolation vers les courtes distances du comportement de $f_{\text {II }}$ (nous raisonnons sur un système de deux particules), l'extrapolation étant faite comme si les particules étaient libres; ainsi $f$ sera une fonction telle que $f \times f$ donne une bonne description des propriétés des deux particules lorsqu'elles sont éloignées, mais «efface » les effets du potentiel à courte distance. Mathématiquement, cette opération sera faite de façon rigoureuse par une modification de la transformée de Wigner en une transformée «libre » qui, tout en donnant moins directement accès aux grandeurs physiques que la véritable transformée de Wigner, se prête mieux à une hypothèse de factorisation (cf. (iv) ci-dessous). 
Quitte à anticiper sur le contenu de cet article et des deux suivants, il nous semble utile pour dégager dans cette introduction une vue d'ensemble de préciser dès maintenant quelques propriétés de cette transformée libre :

(i) elle ne diffère sensiblement de la véritable transformée de Wigner que dans une petite région de l'espace des configurations, celle où la distance relative des particules est de l'ordre, soit de l'ordre de la portée du potentiel, soit de la longueur d'onde de De Broglie.

(ii) Son évolution dans une collision dépend uniquement de paramètres qui s'expriment tous en fonction de la matrice $S$, c'est-à-dire des déphasages, et cela même lorsque les particules sont en cours de collision. Ce ne serait pas le cas de la véritable transformée de Wigner, où subsistent les effets des transitions virtuelles (« off shell terms » de la matrice T) tant que les particules ne se sont pas éloignées. En d'autres termes, l'évolution de $f$ ne dépend que des transitions réelles, où l'énergie cinétique est conservée, tandis que les effets des transitions virtuelles pendant la collision sont concentrés dans $\delta f_{\mathrm{II}}$. Le fait que, pour des particules en train d'interagir, l'évolution de $f$ soit plus simple que celle de $f_{\mathrm{I}}$ est évidemment un point favorable pour l'écriture d'une équation cinétique.

(iii) Un développement systématique permet d'obtenir dans l'équation d'évolution de $f$, en premier lieu les termes habituels ne dépendant que de la section efficace de collision, puis des corrections dues aux effets de retard et de réfraction ; ces corrections font apparaître d'autres paramètres que la section efficace, qui s'expriment toutefois également en fonction de la matrice $S$.

(iv) La transformée de Wigner libre fait apparaître explicitement une partie "entrante » correspondant aux particules qui vont interagir, et une partie «sortante » des particules qui ont interagi. Au cours de la collision, parties entrante et sortante se transforment l'une dans l'autre comme on pouvait s'y attendre ; cependant, à part cette transformation, elles se propagent exactement comme si les particules étaient libres (pas d'effet du potentiel d'interaction).

C'est en fait cette propriété de la transformée libre qui est la propriété cruciale pour toute la suite de ce travail : si, initialement, la partie entrante est factorisée, elle le reste rigoureusement tout au long de l'interaction entre les atomes en collision (effacement des effets du potentiel). On ne fait donc pas d'approximation qui risque de détruire les corrélations à courte distance lorsque l'on suppose une factorisation dans la transformée de Wigner libre. En fait, aucune approximation n'est faite dans le calcul de l'effet d'une collision binaire (comme par exemple de supposer une factorisation de la fonction de distribution à deux corps, ou encore de ne prendre en compte que des collisions terminées comme on le ferait dans le point de vue de Boltzmann) : les relations que nous écrirons au cours de cet article sont complètement exactes, même pendant la collision. De ce point de vue, il est clair que la transformée libre se prête ainsi bien mieux que la véritable fonction $f_{\mathrm{I}}$ à une «Stossansatz » à la Boltzmann et, en conséquence, à l'écriture d'une équation cinétique.

Comme on pouvait s'y attendre cependant, la simplification apportée à l'évolution de la distribution $f$ (comparée à l'évolution de $f_{\mathrm{I}}$ ) est obtenue au prix d'une complication qui apparaît à un stade différent des calculs : ce sont les relations entre les valeurs moyennes locales de grandeurs physiques telles que la densité de particules qui sont moins simples qu'en théorie de Boltzmann; elles deviennent en fait des fonctions non linéaires de $f$.

(v) Une autre propriété commode est son lien étroit avec l'énergie cinétique des particules lorsqu'elles sont éloignées : ainsi apparaîtra très simplement la définition de la température du gaz, alors que cette définition serait plus difficile sur la véritable transformée de Wigner (celle qui donne $f_{\mathrm{I}}$ ).

Dans le présent article (article A), nous définissons donc cette transformée libre, d'abord 
pour une particule unique dans un potentiel, puis pour deux particules en interaction mutuelle. Nous étudions ensuite en détail son évolution dans une collision binaire. L'article suivant (que nous désignerons par B) part des résultats obtenus pour établir une équation cinétique qui généralise l'équation de Boltzmann, tout en respectant les lois de conservation qui sont à la base des équations hydrodynamiques. Ces dernières seront établies et étudiées dans l'article $\mathrm{C}$, où l'on complètera le programme annoncé : obtention des secondes corrections du viriel à partir de l'équation cinétique, et de corrections similaires pour les coefficients de transport.

Précisons à nouveau que seules sont prises en compte dans tout ce travail les collisions binaires ; il ne s'applique donc qu'aux gaz modérément denses, mais pas à ceux qui sont près du point critique par exemple. De plus, pour le moment, nous ne traitons que des particules discernables (pas d'effets de statistique) et sans spin. La généralisation aux effets liés aux spins et à la statistique sera abordée ultérieurement.

\section{Définition de la transformée libre.}

\subsection{PARTICUle DANS UN POTENTIEL.}

1.1.1 Définition. - Considérons une particule (unique, sans spin) d'opérateur densité $\rho$, soumise à l'action d'un potentiel $V$ centré autour de l'origine. Nous noterons $\left|\Psi_{\mathbf{k}}^{(+,-)}\right\rangle$les états stationnaires de diffusion (entrant et sortant) de la particule dans ce potentiel, $\Psi_{\mathbf{k}}^{(+,-)}(\mathbf{r})$ les fonctions d'onde correspondantes et $\Omega^{(+,-)}$les opérateurs unitaires de Möller qui transforment les ondes planes $|\mathbf{k}\rangle$ en ces états stationnaires :

$$
\boldsymbol{\Omega}^{(+,-)}|\mathbf{k}\rangle=\left|\Psi_{\mathbf{k}}^{(+,-)}\right\rangle
$$

Un changement de base à partir de la définition générale de la transformée de Wigner (appendice A, formule (A1)), donne :

$$
\begin{aligned}
\rho_{W}(\mathbf{r}, \mathbf{p})=(2 \pi \hbar)^{-3} \int \mathrm{d}^{3} x \int \mathrm{d}^{3} k_{1} \int \mathrm{d}^{3} k_{2} \mathrm{e}^{i \mathbf{x} \cdot \mathbf{p} / \hbar} \Psi_{\mathbf{k}_{1}}^{(+,-)}\left(\mathbf{r}_{-}\right) & \Psi_{\mathbf{k}_{2}}^{*(+,-)}\left(\mathbf{r}_{+}\right) \times \\
& \times\left\langle\Psi_{\mathbf{k}_{1}}^{(+,-)}|\rho| \Psi_{\mathbf{k}_{2}}^{(+,-)}\right\rangle
\end{aligned}
$$

avec :

$$
\mathbf{r}_{ \pm}=\mathbf{r} \pm \mathbf{x} / 2
$$

Les états stationnaires entrant et sortant sont différents ; cependant si, dans la formule (2), on fait figurer (au choix mais uniformément), soit les ondes entrantes seulement, soit les ondes sortantes, on réobtient la même transformée de Wigner.

Dans la région d'action du potentiel, les fonctions d'onde des états stationnaires de diffusion sont déformées par l'effet du potentiel. Que se produit-il cependant si, dans (2), on efface ces distorsions en remplaçant simplement les $\Psi_{\mathbf{k}}^{(+,-)}(\mathbf{r})$ par des fonctions d'onde non perturbées, les ondes planes $\exp (\mathbf{i k} \cdot \mathbf{r})$ ? Il apparaît alors sous l'intégrale des exponentielles qui rendent possible une sommation immédiate sur $\mathbf{x}$, introduisant ainsi une fonction $\delta$ qui à son tour permet la sommation sur $\mathbf{k}_{1}+\mathbf{k}_{2}$, et donne pour finir :

$$
(2 \pi \hbar)^{-3} \int \mathrm{d}^{3} \kappa \mathrm{e}^{i \kappa \cdot \mathbf{r}}\left\langle\Psi_{\mathbf{k}_{+}}^{(+,-)}|\rho| \Psi_{\mathbf{k}_{-}}^{(+,-)}\right\rangle
$$

avec :

$$
\mathbf{k}_{ \pm}=(\mathbf{p} / \hbar) \pm \kappa / 2
$$


La comparaison avec la dernière des formules (A1) de l'appendice A montre que le résultat ainsi obtenu rappelle fortement une autre expression de la transformée de Wigner ; c'est cependant à la transformée d'un opérateur différent, l'un des opérateurs :

$$
\rho^{\prime}=\left[\Omega^{(+,-)}\right]^{\dagger} \rho\left[\Omega^{(+,-)}\right]
$$

que nous a conduits cette opération de «redressement» des fonctions d'onde. Une telle opération n'a évidemment aucune raison en général de laisser invariante la transformée de Wigner.

Cependant, si l'opérateur densité $\rho$ correspond à une particule qui est encore loin du domaine d'action du potentiel (loin par rapport à la portée du potentiel et également la longueur d'onde de De Broglie), les paquets d'ondes construits à partir des ondes entrantes ou des ondes planes sont les mêmes - c'est dans ce but même que sont construits les états stationnaires entrants ; on peut montrer que, dans le paquet d'ondes, les ondes sphériques sortantes des états stationnaires se détruisent mutuellement par interférence [4] (nous prenons la convention de vocabulaire, fréquente mais pas universelle, où les états stationnaires entrants contiennent une onde sphérique sortante, et vice versa). Dans ce cas, la transformée de Wigner sera aussi bien donnée par (4), sous la version où y figurent les états stationnaires entrants, que par sa définition générale. En d'autres termes, nous prenons dans ce cas un opérateur densité $\rho$ qui commute avec $\Omega^{(+)}$, ce qui permet de montrer facilement que sa transformée de Wigner est donnée par (4). Inversement, il est clair que si $\rho$ décrit une particule qui s'est beaucoup éloignée de la région d'action du potentiel, $\rho$ commute maintenant avec $\Omega^{(-)}$est c'est l'autre version de (4) qu'il faut prendre pour rétablir la vraie transformée de Wigner. Il existe bien sûr un cas, de peu d'intérêt pratique, où les deux versions sont simultanément valables : celui où $\rho$ correspond à une particule qui passe très loin du potentiel et n'interagit jamais avec lui.

La transformée de Wigner libre, que nous allons maintenant introduire, est obtenue par une simple combinaison des deux cas précédents : si le produit scalaire $\mathbf{p} \cdot \mathbf{r}$ est négatif, il s'agit plutôt d'une particule «entrante » et c'est la première modification de la transformée que nous prendrons ; si ce produit scalaire est positif, à l'inverse, nous prendrons la seconde version. Ainsi nous définissons cette transformée libre $\rho_{\mathrm{W}}^{\mathrm{L}}$ par :

$$
\begin{aligned}
\rho_{\mathrm{W}}^{\mathrm{L}}(\mathbf{r}, \mathbf{p})=(2 \pi \hbar)^{-3} \int & \mathrm{d}^{3} \kappa \mathrm{e}^{i \kappa \cdot \mathbf{r}} \times \\
& \times\left\{Y^{(-)}(\mathbf{p} \cdot \mathbf{r})\left\langle\Psi_{\mathbf{k}_{+}}^{(+)}|\rho| \Psi_{\mathbf{k}_{-}}^{(+)}\right\rangle+Y^{(+)}(\mathbf{p} \cdot \mathbf{r})\left\langle\Psi_{\mathbf{k}_{+}}^{(-)}|\rho| \Psi_{\mathbf{k}_{-}}^{(-)}\right\rangle\right\}
\end{aligned}
$$

soit également :

$$
\rho_{\mathrm{W}}^{\mathrm{L}}(\mathbf{r}, \mathbf{p})=Y^{(-)}(\mathbf{p} \cdot \mathbf{r}) \rho_{\mathrm{WL}}^{(+)}(\mathbf{r}, \mathbf{p})+Y^{(+)}(\mathbf{p} \cdot \mathbf{r}) \rho_{\mathrm{WL}}^{(-)}(\mathbf{p}, \mathbf{r})
$$

où apparaissent les deux fonctions, que nous appellerons respectivement «transformée (libre) entrante » et « sortante »:

$$
\left\{\begin{array}{l}
\rho_{\mathrm{WL}}^{(+)}(\mathbf{r}, \mathbf{p})=\text { T.W. }\left\{\left[\Omega^{(+)}\right]^{\dagger} \rho\left[\Omega^{(+)}\right]\right\} \\
\rho_{\mathrm{WL}}^{(-)}(\mathbf{r}, \mathbf{p})=\text { T.W. }\left\{\left[\Omega^{(-)}\right]^{\dagger} \rho\left[\Omega^{(-)}\right]\right\}
\end{array}\right.
$$

Dans ces formules, $Y^{(+)}$désigne la fonction de Heaviside qui vaut 1 si $\mathbf{p} \cdot \mathbf{r}$ est positif, zéro sinon ; inversement pour $Y^{(-)}$; T.W. désigne la transformée de Wigner. Avec cette définition de la transformée de Wigner libre qui combine les deux versions de (4), nous avons maintenant une fonction $\rho$ qui coïncide avec la vraie transformée de Wigner à la fois 
longtemps avant et longtemps après la collision, mais en diffère pendant. Examinons plus en détail quelques propriétés de cette transformée.

1.1.2 Propriétés. - Par construction, la transformée libre diffère de façon essentielle de la transformée de Wigner habituelle dans toute la région d'action du potentiel et, a priori, dans tout un domaine autour de cette région dont la dimension est comparable à la longueur d'onde de De Broglie (dans la plupart des situations, la transformée de Wigner en un point de l'espace ne dépend que des valeurs de la fonction d'onde dans un domaine de cette dimension, mais ce n'est pas une règle absolue ; nous renvoyons aux références citées dans l'appendice $A$ pour une discussion plus détaillée de ces points, qui dépassent le cadre du présent article). Autour de l'origine, elle efface en quelque sorte les effets perturbateurs du potentiel par une extrapolation du comportement à grande distance; le prix de cette simplification est une discontinuité qui se produit dans chaque plan $\mathbf{p} \cdot \mathbf{r}=0$. Sa propagation dans le temps est particulièrement simple à calculer puisque, les états stationnaires de diffusion étant des états propres de l'halmiltonien, on peut directement utiliser dans (7a) les relations :

$$
\left\langle\Psi_{\mathbf{k}_{+}}^{(+,-)}|\rho(t)| \Psi_{\mathbf{k}_{-}}^{(+,-)}\right\rangle=\mathrm{e}^{i\left(\omega_{-}-\omega_{+}\right) t}\left\langle\Psi_{\mathbf{k}_{+}}^{(+,-)}|\rho(0)| \Psi_{\mathbf{k}_{-}}^{(+,-)}\right\rangle
$$

avec :

$$
\omega_{+}-\omega_{-}=\frac{\hbar}{2 m}\left(k_{+}^{2}-k_{-}^{2}\right)=\mathbf{p} \cdot \kappa / m
$$

Ainsi, il suffit d'effectuer dans (7a) la substitution :

$$
\mathbf{r} \Rightarrow \mathbf{r}-\mathbf{p} t / m
$$

(où $m$ est la masse de la particule) pour obtenir la transformée de Wigner libre à un instant quelconque ; dans son évolution, les effets du potentiel ont bien disparu, et elle justifie ainsi son nom de « libre ».

Contrairement à la transformée ordinaire qui est normée - son intégrale sur tout l'espace des phases est égale à la trace de $\rho$, c'est-à-dire 1 - la transformée libre ne satisfait pas à une telle propriété ; la «probabilité manquante » qui s'introduit ainsi sera calculée au début de l'article $B$ et jouera un rôle essentiel dans toute la suite.

Un exemple simple permet de fixer les idées et mettre en lumière les différences entre les deux transformées : celui d'une particule en équilibre thermique dans un potentiel extérieur. L'opérateur densité est alors :

$$
\rho=Z^{-1} \exp \left\{-H / k_{\mathrm{B}} T\right\}
$$

où $Z$ est la fonction de partition, $k_{\mathrm{B}}$ la constante de Boltzmann et $T$ la température; l'hamiltonien est la somme de l'énergie cinétique et de l'énergie potentielle de la particule. L'opérateur densité étant diagonal dans la base des états stationnaires, la formule (7a) se simplifie et donne :

$$
\begin{aligned}
\rho_{\mathrm{W}}^{\mathrm{L}}(\mathbf{r}, \mathbf{p}) & =Z^{-1}(2 \pi \hbar)^{-3} \int \mathrm{d}^{3} \kappa \mathrm{e}^{i \kappa \cdot \mathbf{r}}\left[Y^{(-)}(\mathbf{p} \cdot \mathbf{r})+Y^{(+)}(\mathbf{p} \cdot \mathbf{r})\right] \delta(\kappa) \mathrm{e}^{-p^{2} / 2 m k_{\mathrm{B}} T} \\
& =Z^{-1}(2 \pi \hbar)^{-3} \mathrm{e}^{-p^{2} / 2 m k_{\mathrm{B}} T} .
\end{aligned}
$$

La température libre est donc effectivement une fonction où les effets répulsifs ou attractifs du potentiel ont été « gommés » puisqu'elle est indépendante de $\mathbf{r}$ (elle n'est pas normalisable, 
comme d'ailleurs la véritable transformée de Wigner, tant que la particule n'est pas confinée à un volume fini). Loin du domaine d'action du potentiel, elle coïncide avec la transformée habituelle.

1.2 DEUX PARTICULES EN INTERACTION. - Lorsque deux particules interagissent mutuellement par un potentiel $V$, il est commode de considérer deux particules fictives, celle associée au centre de masse et la « particule relative » qui, seule, est soumise aux effets du potentiel. La transformée de Wigner libre sera alors tout naturellement définie comme la transformée habituelle en ce qui concerne le centre de masse, mais avec le passage en transformée libre comme au paragraphe précédent pour les variables de la particule relative. On posera donc :

$$
\begin{aligned}
& \rho_{\mathrm{W}}^{\mathrm{L}}(\mathbf{R}, \mathbf{r}, \mathbf{P}, \mathbf{p})=(2 \pi \hbar)^{-6} \int \mathrm{d}^{3} \kappa \int \mathrm{d}^{3} K \mathrm{e}^{i \kappa \cdot \mathbf{r}} \mathrm{e}^{i \mathbf{K} \cdot \mathbf{R}} \times \\
& \quad \times\left\{Y^{(-)}(\mathbf{p} \cdot \mathbf{r})\left\langle\mathbf{K}_{+}, \Psi_{\mathbf{k}_{+}}^{(+)}|\rho| \mathbf{K}_{-}, \Psi_{\mathbf{k}_{-}}^{(+)}\right\rangle+Y^{(+)}(\mathbf{p} \cdot \mathbf{r})\left\langle\mathbf{K}_{+}, \Psi_{\mathbf{k}_{+}}^{(-)}|\rho| \mathbf{K}_{-}, \Psi_{\mathbf{k}_{-}}^{(-)}\right\rangle\right\}
\end{aligned}
$$

où $\mathbf{R}$ et $\mathbf{r}$ sont les positions respectives de centre de masse et de la particule relative, $\mathbf{P}$ et $\mathbf{p}$ leurs impulsions :

$$
\begin{array}{ll}
\mathbf{R}=\frac{1}{2}\left(\mathbf{r}_{1}+\mathbf{r}_{2}\right) & \mathbf{r}=\mathbf{r}_{1}-\mathbf{r}_{2} \\
\mathbf{P}=\mathbf{p}_{1}+\mathbf{p}_{2} & \mathbf{p}=\frac{1}{2}\left(\mathbf{p}_{1}-\mathbf{p}_{2}\right) .
\end{array}
$$

Nous supposons pour simplifier que les particules ont même masse, de sorte que la masse totale $M$ et la masse $\mu$ de la particule relative sont simplement données par :

$$
M=2 m \quad \mu=m / 2 .
$$

Pour l'étude d'une collision binaire, qui est notre but pour le moment, la variable d'impulsion qui s'introduit naturellement est $\mathbf{p}$; cependant, dans l'écriture ultérieure d'une équation cinétique, il sera plus commode d'introduire la notation :

$$
\mathbf{q}=\mathbf{p}_{1}-\mathbf{p}_{2}=2 \mathbf{p}
$$

(ainsi, par exemple la trace sur les variables du partenaire de collision correspond-elle simplement à une intégration sur $\mathbf{r}$ et $\mathbf{p}$ dans tout l'espace). Les $\mathbf{k}_{ \pm}$ont été définis en (5); ils s'écrivent également :

$$
\mathbf{k}_{ \pm}=(\mathbf{q} \pm \hbar \kappa) / 2 \hbar
$$

Enfin, les $\mathbf{K}_{ \pm}$sont donnés par :

$$
\mathbf{K}_{ \pm}=\frac{\mathbf{P}}{\hbar} \pm \frac{\mathbf{K}}{2}
$$

La définition (11) est donc la généralisation de (7a) au cas de deux particules en interaction ; les définitions (7c) des transformées « entrante » et « sortante » restent valables (compte tenu du fait que l'opérateur $\Omega$ n'agit que dans l'espace des états de la particule relative, mais pas dans celui du centre de masse). Ainsi que précédemment, ces transformées libres se propagent comme le feraient des transformées ordinaires en l'absence d'interaction, ce qui conduit à effectuer les substitutions :

$$
\mathbf{r}_{1,2} \Rightarrow \mathbf{r}_{1,2}-\mathbf{p}_{1,2} t / m \text {. }
$$


On a donc la nullité des « dérivées totales » :

$$
\left[\frac{\partial}{\partial t}+\sum_{i=1}^{2} \frac{\mathbf{p}_{i}}{m} \cdot \nabla_{\mathbf{r}_{i}}\right] \rho_{\mathrm{WL}}^{(+,-)}=0 .
$$

Il sera utile pour la suite d'inverser les relations (7c) de transformation de Wigner (Appendice A, relation (A6)) et d'écrire :

$$
\begin{aligned}
& \left\langle\mathbf{K}_{1}, \mathbf{k}_{1}\left|\left[\Omega^{(+,-)}\right]^{\dagger} \rho\left[\Omega^{(+,-)}\right]\right| \mathbf{K}_{2}, \mathbf{k}_{2}\right\rangle=\left\langle\mathbf{K}_{1}, \Psi_{\mathbf{k}_{1}}^{(+,-)}|\rho| \mathbf{K}_{2}, \Psi_{\mathbf{k}_{2}}^{(+,-)}\right\rangle= \\
& \quad=\hbar^{6} \int \mathrm{d}^{3} R^{\prime} \int \mathrm{d}^{3} r^{\prime} \mathrm{e}^{i\left(\mathbf{K}_{2}-\mathbf{K}_{1}\right) \cdot \mathbf{R}^{\prime}} \mathrm{e}^{i\left(\mathbf{k}_{2}, \mathbf{k}_{1}\right) \cdot \mathbf{r}^{\prime}} \rho_{\mathrm{WL}^{+}}^{(-)}\left(\mathbf{R}^{\prime}, \mathbf{r}^{\prime}, \hbar \frac{\mathbf{K}_{1}+\mathbf{K}_{2}}{2}, \hbar \frac{\mathbf{k}_{1}+\mathbf{k}_{2}}{2}\right) .
\end{aligned}
$$

\section{Evolution dans une collision binaire.}

La définition (11) se prête facilement au calcul de l'évolution de la transformée libre, puisqu'y figurent directement les éléments de matrice de l'opérateur densité entre des états propres de l'énergie, dont les fréquences propres sont données par:

$$
\begin{aligned}
\hbar\left[k_{+}^{2}-k_{-}^{2}\right] / 2 \mu & =\mathbf{p} \cdot \kappa / \mu \\
\hbar\left[K_{+}^{2}-K_{-}^{2}\right] / 2 M & =\mathbf{P} \cdot \mathbf{K} / M .
\end{aligned}
$$

Nous allons cependant faire ici un calcul différent, basé seulement sur les relations unitaires qui lient les opérateurs qui apparaissent aux seconds membres de $(7 \mathrm{c})$, et dont $\rho_{\mathrm{WL}}^{(+)}$et $\rho_{\mathrm{WL}}^{(-)}$sont les transformées de Wigner; notre but est d'exprimer entièrement l'évolution de ces deux fonctions à partir de la seule transformée « libre entrante ». Injectons donc, de chaque côté de l'opérateur $\rho$ qui figure dans la partie «sortante » du second membre de (11), une relation de fermeture sur les états stationnaires entrants; il vient alors l'intégrale :

$$
\begin{aligned}
(2 \pi \hbar)^{-6} \int \mathrm{d}^{3} \kappa \int \mathrm{d}^{3} K \int \mathrm{d}^{3} k_{1} \int \mathrm{d}^{3} k_{2} \mathrm{e}^{i \kappa \cdot \mathbf{r}} \mathrm{e}^{i \mathbf{K} \cdot \mathbf{R}} \times \\
\quad \times S\left(\mathbf{k}_{+}, \mathbf{k}_{1}\right) S^{*}\left(\mathbf{k}_{-}, \mathbf{k}_{2}\right)\left\langle\mathbf{K}_{+}, \Psi_{\mathbf{k}_{+}}^{(+)}|\rho| \mathbf{K}_{-}, \Psi_{\mathbf{k}_{-}}^{(+)}\right\rangle
\end{aligned}
$$

avec la notation usuelle :

$$
S\left(\mathbf{k}^{\prime}, \mathbf{k}\right)=\left\langle\Psi_{\mathbf{k}^{\prime}}^{(-)} \mid \Psi_{\mathbf{k}}^{(+)}\right\rangle=\left\langle\mathbf{k}^{\prime}\left|\left[\Omega^{(-)}\right]^{\dagger}\left[\Omega^{(+)}\right]\right| \mathbf{k}\right\rangle .
$$

Mais nous pouvons alors utiliser l'égalité (14) pour exprimer l'élément de matrice de $\rho$ en fonction de la transformée entrante $\rho_{\mathrm{WL}}^{(+)}$; il apparaît alors l'intégrale :

$$
\int \mathrm{d}^{3} K \mathrm{e}^{i \mathbf{K} \cdot\left(\mathbf{R}-\mathbf{R}^{\prime}\right)}=(2 \pi)^{3} \delta\left(\mathbf{R}-\mathbf{R}^{\prime}\right)
$$

qui permet d'éliminer la sommation sur $\mathrm{d}^{3} R^{\prime}$, de sorte que, pour finir :

$$
\begin{aligned}
\rho_{\mathrm{WL}}^{(-)}(\mathbf{R}, \mathbf{r}, \mathbf{P}, \mathbf{p})=(2 \pi)^{-3} \int \mathrm{d}^{3} \kappa & \int \mathrm{d}^{3} k_{1} \int \mathrm{d}^{3} k_{2} \int \mathrm{d}^{3} r^{\prime} \mathrm{e}^{i \kappa \cdot \mathbf{r}} \mathrm{e}^{i\left(\mathbf{k}_{2}-\mathbf{k}_{1}\right) \cdot \mathbf{r}^{\prime}} \times \\
& \times S\left(\mathbf{k}_{+}, \mathbf{k}_{1}\right) S^{*}\left(\mathbf{k}_{-}, \mathbf{k}_{2}\right) \rho_{\mathrm{WL}}^{(+)}\left(\mathbf{R}, \mathbf{r}^{\prime}, \mathbf{P}, \hbar \frac{\mathbf{k}_{1}+\mathbf{k}_{2}}{2}\right) .
\end{aligned}
$$


Cette relation est simplement celle d'une transformée unitaire (Appendice A, formule (8)), sous l'action de l'opérateur $\left[\Omega^{(-)}\right]^{\dagger}\left[\Omega^{(+)}\right]$qui n'agit que dans l'espace des états de la particule relative, d'où le fait qu'aucune convolution n'affecte les variables associées au centre de masse. Quant à l'expression de la transformée libre dans son ensemble, elle est :

$$
\begin{aligned}
\rho_{\mathrm{W}}^{\mathrm{L}}(\mathbf{R}, \mathbf{r}, \mathbf{P}, \mathbf{p})=(2 \pi)^{-3} \int \mathrm{d}^{3} \kappa \int \mathrm{d}^{3} k_{1} \int \mathrm{d}^{3} k_{2} \int \mathrm{d}^{3} r^{\prime} \mathrm{e}^{i \kappa \cdot \mathbf{r}} \mathrm{e}^{i\left(\mathbf{k}_{2}-\mathbf{k}_{1}\right) \cdot \mathbf{r}^{\prime}} \times \\
\quad \times\left[Y^{(+)}(\mathbf{p} \cdot \mathbf{r}) S\left(\mathbf{k}_{+}, \mathbf{k}_{1}\right) S^{*}\left(\mathbf{k}_{-}, \mathbf{k}_{2}\right)+Y^{(-)}(\mathbf{p} \cdot \mathbf{r}) \delta\left(\mathbf{k}_{+}-\mathbf{k}_{1}\right) \delta\left(\mathbf{k}_{-}-\mathbf{k}_{2}\right)\right] \\
\quad \times \rho_{\mathrm{WL}}^{(+)}\left(\mathbf{R}, \mathbf{r}^{\prime}, \mathbf{P}, \hbar \frac{\mathbf{k}_{1}+\mathbf{k}_{2}}{2}\right)
\end{aligned}
$$

(il est aisé de vérifier que le terme qui contient deux fonctions delta sous l'intégrale reconstruit la partie entrante), expression qui sera notre point de départ pour le calcul de l'évolution de la transformée de Wigner libre.

Plus précisément, nous allons calculer la « dérivée totale » :

$$
\left[\frac{\partial}{\partial t}+\sum_{i=1}^{2} \frac{\mathbf{p}_{i}}{m} \cdot \nabla_{\mathbf{r}_{i}}\right] \rho_{\mathrm{W}}^{\mathrm{L}}\left(\mathbf{r}_{1}, \mathbf{r}_{2}, \mathbf{p}_{1}, \mathbf{p}_{2}\right)
$$

(nous considérerons indifféremment les transformées de Wigner, libre ou non, comme des fonctions des variables individuelles des particules ou de celles des particules relatives et centre de masse ; compte tenu des relations opératorielles (12a), il est facile de montrer que les transformations de Wigner par rapport aux deux types de variables conduisent à des fonctions qui sont en fait égales, et nous ne les distinguerons pas formellement). On sait que :

$$
\sum_{i} \frac{\mathbf{p}_{i}}{m} \cdot \nabla_{\mathbf{r}_{i}}=\frac{\mathbf{P}}{M} \cdot \nabla_{\mathbf{R}}+\frac{\mathbf{p}}{\mu} \cdot \nabla_{\mathbf{r}} .
$$

Laissons pour le moment de côté le terme de dérivation pour la particule centre de masse, que nous regrouperons avec la dérivation temporelle, et étudions l'effet sur (20) du terme de gradient par rapport à $\mathbf{r}$. Deux sortes de contributions sont à prendre en compte : la première provient de la dérivation des fonctions de Heaviside, qui donne immédiatement l'intégrale :

$$
\begin{aligned}
I_{\mathrm{c}}=( & (2 \pi)^{-3} \frac{p}{\mu} \delta(\hat{\mathbf{p}} \cdot \mathbf{r}) \int \mathrm{d}^{3} \kappa \int \mathrm{d}^{3} k_{1} \int \mathrm{d}^{3} k_{2} \int \mathrm{d}^{3} r^{\prime} \mathrm{e}^{i \kappa \cdot \mathbf{r}} \mathrm{e}^{i\left(\mathbf{k}_{2}-\mathbf{k}_{1}\right) \cdot \mathbf{r}^{\prime}} \times \\
& \times\left[S\left(\mathbf{k}_{+}, \mathbf{k}_{1}\right) S^{*}\left(\mathbf{k}_{-}, \mathbf{k}_{2}\right)-\delta\left(\mathbf{k}_{+}-\mathbf{k}_{1}\right) \delta\left(\mathbf{k}_{-}-\mathbf{k}_{2}\right)\right] \rho_{\mathrm{WL}}^{(+)}\left(\mathbf{R}, \mathbf{r}^{\prime}, \mathbf{P}, \hbar \frac{\mathbf{k}_{1}+\mathbf{k}_{2}}{2}\right)
\end{aligned}
$$

où $\hat{\mathbf{p}}$ est le vecteur unitaire :

$$
\hat{\mathbf{p}}=\mathbf{p} / p
$$

et la seconde de la dérivation de l'exponentielle $\mathrm{e}^{i \kappa \cdot r}$. Pour cette dernière s'introduit sous l'intégrale l'expression écrite en (15a), multipliée par $i$; mais, comme les éléments de la matrice $S$ qui interviennent dans l'intégrale (20) conservent l'énergie (ils contiennent une fonction $\delta\left(k-k^{\prime}\right)$, cf. (33)), ce coefficient peut être remplacé par :

$$
i \frac{\hbar}{2 \mu}\left[k_{1}^{2}-k_{2}^{2}\right]=i \frac{\hbar}{2 \mu}\left(\mathbf{k}_{1}+\mathbf{k}_{2}\right) \cdot\left(\mathbf{k}_{1}-\mathbf{k}_{2}\right) \text {. }
$$


On peut alors absorber le coefficient $\left(\mathbf{k}_{1}-\mathbf{k}_{2}\right)$ dans une dérivation d'une exponentielle par rapport à $\mathbf{r}^{\prime}$ selon :

$$
i \nabla_{\mathbf{r}^{\prime}} \mathrm{e}^{i\left(\mathbf{k}_{2}-\mathbf{k}_{1}\right) \cdot \mathbf{r}^{\prime}}
$$

et, par intégration par parties sur cette variable, faire apparaître la quantité :

$$
\frac{\hbar}{2 \mu}\left(\mathbf{k}_{1}+\mathbf{k}_{2}\right) \cdot \nabla_{\mathbf{r}^{\prime}} \rho_{\mathrm{WL}}^{(+)}\left(\mathbf{R}, \mathbf{r}^{\prime}, \mathbf{P}, \hbar \frac{\mathbf{k}_{1}+\mathbf{k}_{2}}{2}\right) \text {. }
$$

Il ne reste maintenant qu'à regrouper le terme ainsi obtenu avec ceux, laissés de côté, de dérivation par rapport à $\mathbf{R}$ et à $t$ pour obtenir [compte tenu à nouveau de (21b)] un terme qui contient sous l'intégrale l'expression :

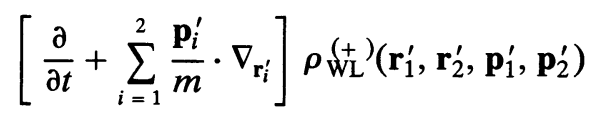

avec la notation :

$$
\left\{\begin{array}{l}
\mathbf{r}_{1,2}^{\prime}=\mathbf{R} \pm \frac{\mathbf{r}^{\prime}}{2} \\
\mathbf{p}_{1,2}^{\prime}=\frac{\mathbf{P}}{2} \pm \hbar \frac{\mathbf{k}_{1}+\mathbf{k}_{2}}{2}
\end{array}\right.
$$

En résumé, si nous notons $D_{\mathrm{T}}$ la dérivée totale :

$$
D_{\mathrm{T}}=\left[\frac{\partial}{\partial t}+\sum_{i=1}^{2} \frac{\mathbf{p}_{i}}{m} \cdot \nabla_{\mathbf{r}_{i}}\right]
$$

nous avons donc:

$$
D_{\mathrm{T}} \rho_{\mathrm{W}}^{\mathrm{L}}\left(\mathbf{r}_{1}, \mathbf{r}_{2}, \mathbf{p}_{1}, \mathbf{p}_{2} ; t\right)=I_{\mathrm{c}}+I_{\mathrm{c}}^{\prime}
$$

où $I_{\mathrm{c}}$ a été défini en (22) et où $I_{\mathrm{c}}^{\prime}$ est donné par :

$$
\begin{aligned}
I_{\mathrm{c}}^{\prime}=(2 \pi)^{-3} & \int \mathrm{d}^{3} \kappa \int \mathrm{d}^{3} k_{1} \int \mathrm{d}^{3} k_{2} \int \mathrm{d}^{3} r^{\prime} \times \\
& \times\left[S\left(\mathbf{k}_{+}, \mathbf{k}_{1}\right) S^{*}\left(\mathbf{k}_{-}, \mathbf{k}_{2}\right)+\delta\left(\mathbf{k}_{+}-\mathbf{k}_{1}\right) \delta\left(\mathbf{k}_{-}-\mathbf{k}_{2}\right)\right] D_{\mathrm{T}}^{\prime} \rho_{\mathrm{WL}}^{(+)}\left(\mathbf{r}_{1}^{\prime}, \mathbf{r}_{2}^{\prime}, \mathbf{p}_{1}^{\prime}, \mathbf{p}_{2}^{\prime}\right)
\end{aligned}
$$

où $D_{\mathrm{T}}^{\prime}$ est l'opérateur différentiel (29) par rapport aux variables (28). L'égalité (30), jointe aux définitions (22) et (31) de $I_{\mathrm{c}}$ et $I_{\mathrm{c}}^{\prime}$, est une conséquence tout à fait générale de (20); cette relation peut être considérée comme la définition de la transformée de Wigner libre, quelle que soit l'évolution de l'opérateur densité $\rho$. Cependant, on peut évidemment introduire la dynamique des deux particules en interaction par l'intermédiaire des relations (13), qui entraînent la nullité de l'intégrale $I_{\mathrm{c}}^{\prime}$ et aussitôt :

$$
D_{\mathrm{T}} \rho_{\mathrm{W}}^{\mathrm{L}}=I_{\mathrm{c}}
$$

relation qui est donc valable pour deux particules sans autre interaction que leur interaction mutuelle.

Ainsi, contrairement à la véritable transformée de Wigner, la transformée libre évolue 
selon une équation qui fait seulement intervenir les éléments de la matrice $S$, qui est ellemême reliée à la matrice $T$, par :

$$
S\left(\mathbf{k}^{\prime}, \mathbf{k}\right)=\delta\left(\mathbf{k}^{\prime}-\mathbf{k}\right)-2 i \pi \frac{\mu}{\hbar^{2} k} \delta\left(k^{\prime}-k\right) T\left(\mathbf{k}^{\prime}, \mathbf{k}\right)
$$

ce qui implique que seuls jouent un rôle pour l'évolution les éléments de $T$ « sur la couche de masse ».

Comme nous étudions dans cet article un système de deux particules isolées, nous allons ignorer $I_{\mathrm{c}}^{\prime}$ et étudier en détail la structure de l'intégrale de collision $I_{\mathrm{c}}$ (l'intégrale $I_{\mathrm{c}}^{\prime}$ jouera un rôle à partir de l'article B). Après insertion de (33) dans (22), il apparaît trois sortes de termes: ceux de degré zéro en $T$, qui disparaissent immédiatement par compensation avec les fonctions delta dans le crochet (on retrouve ainsi qu'en l'absence d'interaction le terme de collision est bien nul); deux termes linéaires en $T$, qui correspondent aux effets de «diffusion vers l'avant» (interférence entre l'onde plane incidente et l'onde diffusée sphérique sortante); enfin les termes quadratiques en $T$ qui traduisent les effets de diffusion latérale. La suite de cet article est consacrée au calcul de ces termes.

\section{Termes habituels de collision.}

3.1 IDÉE GÉNÉRALE DU CALCUL. - Les équations (22) et (32) nous montrent que l'évolution de la transformée libre se fait de façon «non locale », puisque les variations en chaque point $\mathbf{r}$ dépendent des valeurs de la partie entrante en des points $\mathbf{r}^{\prime}$ différents. Il n'y a là rien d'étonnant, on trouvera dans les références [5-10] d'autres exemples d'intégrales de collision possédant la même propriété. La non-localité de (22) prend la forme d'une intégrale de convolution, dont le noyau est lui-même une intégrale multiple. Cependant, dans cette intégrale n'interviennent que des quantités microscopiques ; c'est dire que l'influence d'un point $\mathbf{r}^{\prime}$ sur un point $\mathbf{r}$ a une portée de l'ordre, soit de la portée du potentiel (contenue dans les propriétés de la matrice $S$ ), soit de la longueur d'onde de De Broglie. Il est donc utile pour la suite de s'intéresser aux variations spatiales de la partie entrante à cette échelle.

De façon générale, on sait (Refs. [5-8]) que la transformée de Wigner peut présenter des comportements compliqués, osciller ou même devenir négative dans de petites régions de l'espace des phases; ces comportements disparaissent cependant par moyennage dans l'espace des phases sur des volumes grands par rapport à $\hbar^{3}$. Cependant, dans le présent travail, nous pouvons supposer que les variations spatiales de la transformée de Wigner initiale (avant collision) sont lentes à cette échelle. Cela n'exclut en rien la possibilité que, pendant la collision, des variations spatiales rapides, d'échelle microscopique, se créent sous l'effet du potentiel : nous supposons seulement qu'elles n'existent pas avant le début de la collision. Dans ces conditions, comme la transformée de Wigner libre entrante coïncide initialement avec la véritable transformée de Wigner, et qu'elle se propage de la même façon qu'en l'absence de potentiel, elle conserve nécessairement des variations spatiales lentes, même pendant collision.

Ainsi, un développement de $\rho_{\mathrm{WL}}^{(+)}$en gradients spatiaux dans le second membre de (22) estil naturel, et devrait-il converger rapidement. C'est l'objet des paragraphes qui suivent : le terme d'ordre zéro en gradients sera évalué dans ce paragraphe 3 , et nous verrons qu'il engendre simplement le terme de collision habituel de Boltzmann; le terme d'ordre suivant introduit des corrections qui seront calculées au paragraphe 4. Sur le plan mathématique, il s'agit d'obtenir la transformée de Wigner d'un produit de trois opérateurs. C'est donc la généralisation de la formule de Groenewold [15], valable pour un produit de deux opérateurs, 
et qui contient successivement les produits des transformées de Wigner, leur crochet de Poisson, et une série infinie de termes contenant des dérivées d'ordre croissant; nous obtiendrons effectivement des résultats du même type.

Une hypothèse de variation spatiale lente à échelle microscopique de la transformée de Wigner avant collision est évidemment très largement vérifiée si l'on s'intéresse à des expériences de collision jet sur jet en physique atomique. C'est le point de vue que nous prenons dans cet article. Mais, anticipons pour un instant avec le point de vue qui sera adopté à partir de l'article $\mathrm{B}$, où nous nous intéresserons à un grand nombre d'atomes dans un gaz hors d'équilibre ; l'hypothèse de variation spatiale lente de la transformée libre reviendra à supposer que la fonction de distribution à une particule $f_{\mathrm{I}}$ - et donc les grandeurs hydrodynamiques comme par exemple la vitesse moyenne locale - ne varie pas à une échelle microscopique. Elle n'implique en rien que la fonction de distribution $f_{\text {II }}$ aie la même propriété : car elle peut parfaitement varier de façon significative en fonction de la variable $\mathbf{r}$ à l'échelle de la portée du potentiel ; en revanche, ces variations rapides ne se produisent pas pour la dépendance en fonction de $\mathbf{R}$, ce qui explique leur disparition de $f_{\mathrm{I}}$ par moyennage spatial. Incidemment, une telle hypothèse ne nous restreindra pas à l'étude de situations proches de l'équilibre pour la distribution de Boltzmann : nous n'excluons pas des variations rapides de $f_{\mathrm{I}}$ (et de $f$ ) à l'échelle du libre parcours moyen dans le gaz.

3.2 TERMES DE DIFFUSION VERS L'AVANT. - Il existe deux termes linéaires en $T$ dans (22), l'un proportionnel à $T$, l'autre à $T^{*}$ qui est complexe conjugué du précédent. Calculons par exemple le premier d'entre eux; sous l'intégrale, on peut remplacer $\mathbf{k}_{2}$ par $\mathbf{k}_{-}$et $\mathbf{k}_{1}$ par $k_{+} \hat{\mathbf{k}}$, où $\hat{\mathbf{k}}$ est le vecteur unitaire de même direction que $\mathbf{k}_{1}$; on obtient ainsi (nous prenons un axe $\mathrm{O} z$ parallèle à $\mathbf{p})$ :

$$
\begin{aligned}
-i(2 \pi \hbar)^{-2} p \delta(z) \int \mathrm{d}^{3} \kappa \int \mathrm{d}^{2} \hat{k} \int & \mathrm{d}^{3} r^{\prime} \mathrm{e}^{i \kappa \cdot \mathbf{r}} \mathrm{e}^{i\left(\mathbf{k}_{-}-k_{+} \hat{\mathbf{k}}\right) \cdot \mathbf{r}^{\prime}} \times \\
& \times k_{+} T\left(\mathbf{k}_{+}, k_{+} \hat{\mathbf{k}}\right) \rho_{\mathrm{WL}}^{(+)}\left(\mathbf{R}, \mathbf{r}^{\prime}, \mathbf{P}, \hbar \frac{k_{+} \hat{\mathbf{k}}+\mathbf{k}_{-}}{2}\right) .
\end{aligned}
$$

Nous allons nous intéresser à la trace sur la particule 2 de ce terme ; pour le moment, nous n'effectuerons que la sommation sur $\mathbf{r}_{2}$. En fait, il sera plus commode de prendre comme variable d'intégration la position $\mathbf{r}$ de la particule relative, ce qui conduit à effectuer dans (34) la substitution :

$$
\mathbf{R} \Rightarrow \mathbf{r}_{1}-\mathbf{r} / 2
$$

La sommation sur $z$ est immédiate à cause de la présence de la fonction $\delta$; elle permet de remplacer $\mathrm{e}^{i \kappa \cdot \mathbf{r}}$ par :

$$
\mathrm{e}^{i \kappa_{x} x} \mathrm{e}^{i \kappa_{y} y}
$$

Pour effectuer celle sur $x$ et $y$, nous développons $\rho_{\mathrm{WL}}^{(+)}$suivant :

$$
\left.\left.\rho_{\mathrm{WL}}^{(+)}\right)\left(\mathbf{R}=\mathbf{r}_{1}-\mathbf{r} / 2, \ldots\right)=\rho_{\mathrm{WL}}^{(+)}\left(\mathbf{R}=\mathbf{r}_{1}, \ldots\right)-\frac{\mathbf{r}}{2} \cdot \nabla_{\mathbf{R}} \rho_{\mathrm{WL}}^{(++}\right)\left(\mathbf{R}=\mathbf{r}_{1}, \ldots\right)+\ldots
$$

et nous ne gardons pour le moment que le premier terme du développement (ordre zéro). Alors, les seules dépendances en $x$ et $y$ de la fonction à intégrer sont contenues dans les exponentielles de (36), de sorte que les sommations sur $x$ et $y$ introduisent des fonctions delta 
des composantes sur $\mathrm{O} x$ et $\mathrm{O} y$ de $\kappa$; notant pour simplifier $\kappa$ celle sur $\mathrm{O} z$, nous obtenons ainsi :

$$
\begin{aligned}
-\frac{i p}{\hbar^{2}} \int \mathrm{d} \kappa \int \mathrm{d}^{2} \hat{k} \int \mathrm{d}^{3} r^{\prime} \mathrm{e}^{\left(k_{-} \hat{\mathbf{p}}-k_{+} \hat{\mathbf{k}}\right) \cdot \mathbf{r}^{\prime}} \times \\
\quad \times k_{+} T\left(\tilde{k}_{+} \hat{\mathbf{p}}, k_{+} \hat{\mathbf{k}}\right) \rho_{\mathrm{WL}}^{(+)}\left(\mathbf{R}=\mathbf{r}_{1}, \mathbf{r}^{\prime}, \mathbf{P}, \hbar \frac{k_{+} \hat{\mathbf{k}}+\tilde{k}_{-} \hat{\mathbf{p}}}{2}\right)
\end{aligned}
$$

où $\hat{\mathbf{p}}$ est le vecteur unitaire de $\mathbf{p}$, parallèle à $\mathrm{O} z$, et $\tilde{k}_{ \pm}$désigne la composante de $\mathbf{k}_{ \pm} \operatorname{sur} \hat{\mathbf{p}}$ (qui vaut, soit $k_{ \pm}$, soit son opposé, suivant la valeur de $\kappa$ ).

Passons maintenant des deux variables angulaires du vecteur unitaire $\hat{\mathbf{k}}$ aux deux composantes cartésiennes $\hat{k}_{x}$ et $\hat{k}_{y}$ de $\hat{\mathbf{k}}$ sur $\mathrm{O} x$ et $\mathrm{O} y$; cette opération introduit (le module d') un jacobien qui vaut $\cos \theta$, où $\theta$ est l'angle entre $\hat{\mathbf{k}}$ et $\mathrm{O} z$, et conduit à une intégration sur deux nappes puisque :

$$
\hat{k}_{z}= \pm \sqrt{1-\hat{k}_{x}^{2}-\hat{k}_{y}^{2}}
$$

Alors :

$$
\mathrm{e}^{i\left(\tilde{k}_{-} \mathbf{p}-k_{+} \hat{\mathbf{k}}\right) \cdot \mathbf{r}^{\prime}}=\mathrm{e}^{-i k_{+}\left[k_{x} x^{\prime}+k_{y} y^{\prime}\right]} \mathrm{e}^{i\left(\tilde{k}_{-}-k_{+} k_{z}\right) z^{\prime}}
$$

Pour effectuer l'intégration sur $x^{\prime}$ et $y^{\prime}$, développons encore la dépendance spatiale de $\rho_{\mathrm{WL}}^{(+)}$suivant :

$$
\rho_{\mathrm{WL}}^{(+)}\left(\ldots, \mathbf{r}^{\prime}, \ldots\right)=\rho_{\mathrm{WL}}^{(+)}(\ldots, \mathbf{0}, \ldots)+\mathbf{r}^{\prime} \cdot \nabla_{\mathbf{r}^{\prime}} \rho_{\mathrm{WL}}^{(+)}(\ldots, \mathbf{0}, \ldots)+\ldots
$$

A nouveau, nous nous limitons pour le moment au terme d'ordre zéro en gradients. Dans ces conditions, par sommation sur $x^{\prime}$ et $y^{\prime}$ de l'exponentielle (40) apparaissent des fonctions delta des composantes sur $\mathrm{O} x$ et $\mathrm{O} y$ de $k_{+} \hat{\mathbf{k}}$ :

$$
\int \mathrm{d} x^{\prime} \int \mathrm{d} y^{\prime} \mathrm{e}^{-i k_{+}\left[k_{x} x^{\prime}+k_{y} y^{\prime}\right]}=4 \pi^{2} \delta\left(k_{+} \hat{k}_{x}\right) \delta\left(k_{+} \hat{k}_{y}\right)
$$

de sorte que, dans cette exponentielle, on peut reporter :

$$
k_{+} \hat{k}_{z}= \pm k_{+}
$$

Examinons successivement les contributions de ces deux déterminations. Pour la première, la sommation sur $z^{\prime}$ introduit l'intégrale :

$$
\int \mathrm{d} z^{\prime} \mathrm{e}^{i\left(\tilde{k}_{-}-k_{+}\right) z^{\prime}}=2 \pi \delta\left(k_{+}-\tilde{k}_{-}\right)=2 \pi \delta(\kappa)
$$

(le module du vecteur $\mathbf{k}_{+}$et le nombre algébrique $\tilde{k}_{-}$ne peuvent être égaux que si $\kappa$ s'annule, comme on peut s'en assurer aisément); on peut alors simplifier (42b) par la quantité $k_{+}$non nulle et obtenir, pour cette première nappe :

$$
\hat{\mathbf{k}}=+\hat{\mathbf{p}}
$$


(qui impose la valeur un au jacobien). L'intégration sur $\hat{k}_{x}$ et $\hat{k}_{y}$ est alors simple puisque la non-annulation de $k_{+}$permet de réécrire le second membre de (42a) sous la forme :

$$
\left(2 \pi / k_{+}\right)^{2} \delta\left(\hat{k}_{x}\right) \delta\left(\hat{k}_{y}\right)
$$

et il vient :

$$
\left.-8\left(i \pi^{3} / \hbar\right) T_{p / \hbar}(1) \rho_{\mathrm{WL}}^{(+)}\right)\left(\mathbf{R}=\mathbf{r}_{1}, \mathbf{0}, \mathbf{P}, \mathbf{p}\right)
$$

(par un léger changement de notation, nous notons maintenant la dépendance de $T$ en $p / \hbar$ et en cosinus de l'angle de collision). Pour la seconde détermination de (42b), elle introduit une fonction delta de la somme du module du vecteur $\mathbf{k}_{+}$et de $\tilde{k}_{-}$, qui n'est jamais nulle, de sorte qu'elle ne contribue pas au résultat.

Regroupant maintenant (44) avec le terme complexe conjugué introduit par $T^{*}$, nous obtenons pour finir :

$$
-\frac{p}{\mu} \sigma_{T}(p) \rho_{\mathrm{WL}}^{(+)}\left(\mathbf{R}=\mathbf{r}_{1}, \mathbf{r}=\mathbf{0}, \mathbf{P}, \mathbf{p}\right)
$$

où l'on retrouve la définition habituelle de la section efficace totale (théorème optique) :

$$
\sigma_{T}(p)=-\frac{16 \pi^{3} \mu}{\hbar p} \operatorname{Im}\left\{T_{p / \hbar}(1)\right\} .
$$

L'interprétation physique de ce terme est simple : à cet ordre en gradients, les termes linéaires en $T$ font simplement apparaître les termes de « départ » dans l'intégrale de collision de Boltzmann (sans l'intégrale triple sur l'impulsion puisque, si nous avons déjà sommé sur $\mathbf{r}_{2}$, nous n'avons pas encore sommé sur l'impulsion de la seconde particule). Aucun raisonnement semi-classique n'a été nécessaire pour introduire, ni la section efficace, ni le préfacteur $p / \mu$, dont le produit est habituellement introduit comme le « volume balayé » par la particule ; ils sont conséquences du raisonnement général effectué sur la transformée de Wigner libre.

3.3 TERMES DE DiffuSiOn latérale. - Le terme quadratique en $T$ issu de (22) s'écrit :

$$
\begin{aligned}
\left(p^{2} \mu / 2 \pi \hbar^{4}\right) & \delta(\hat{\mathbf{p}} \cdot \mathbf{r}) \int \mathrm{d}^{3} \kappa \int \mathrm{d}^{2} \hat{k}_{1} \int \mathrm{d}^{2} \hat{k}_{2} \int \mathrm{d}^{3} r^{\prime} \mathrm{e}^{i \kappa \cdot \mathbf{r}} \mathrm{e}^{i\left(k_{-} \hat{\mathbf{k}}_{2}-k_{+} \hat{\mathbf{k}}_{1}\right) \cdot \mathbf{r}^{\prime}} \times \\
& \times k_{+} k_{-} T\left(\mathbf{k}_{+}, k_{+} \hat{\mathbf{k}}_{1}\right) T^{*}\left(\mathbf{k}_{-}, k_{-} \hat{\mathbf{k}}_{2}\right) \rho_{\mathrm{WL}}^{(+)}\left(\mathbf{R}, \mathbf{r}^{\prime}, \mathbf{P}, \hbar \frac{k_{+} \hat{\mathbf{k}}_{1}+k_{-} \hat{\mathbf{k}}_{2}}{2}\right) .
\end{aligned}
$$

Comme plus haut, nous effectuons la trace sur la position de la particule 2 en prenant $\mathbf{r}$ comme variable d'intégration et en effectuant la substitution (35). Nous développons de même la dépendance de $\rho_{\mathrm{WL}}^{(+)}$en $\mathbf{R}$ (formule (37)) ce qui, à l'ordre zéro, nous permet à nouveau de faire apparaître des fonctions delta des composantes de $\kappa$ sur le plan perpendiculaire à $\mathbf{p}$ et d'obtenir :

$$
\begin{aligned}
& \left(2 \pi p \mu / h^{4}\right) \int \mathrm{d} \kappa \int \mathrm{d}^{2} \hat{k}_{1} \int \mathrm{d}^{2} \hat{k}_{2} \int \mathrm{d}^{3} r^{\prime} \mathrm{e}^{i\left(k_{-} \hat{\mathbf{k}}_{2}-k_{+} \hat{\mathbf{k}}_{1}\right) \cdot \mathbf{r}^{\prime}} \times \\
& \quad \times k_{+} k_{-} T\left(\tilde{k}_{+} \hat{\mathbf{p}}, k_{+} \hat{\mathbf{k}}_{1}\right) T^{*}\left(\tilde{k}_{-} \hat{\mathbf{p}}, k_{-} \hat{\mathbf{k}}_{2}\right) \rho_{\mathrm{WL}}^{(+)}\left(\mathbf{R}=\mathbf{r}_{1}, \mathbf{r}^{\prime}, \mathbf{P}, \hbar \frac{k_{+} \hat{\mathbf{k}}_{1}+k_{-} \hat{\mathbf{k}}_{2}}{2}\right) .
\end{aligned}
$$


Nous prenons maintenant (ce sera le cas chaque fois que nous calculerons des termes de diffusion latérale) l'axe $\mathrm{O} z$ parallèle, non pas à $\mathbf{p}$, mais à $\mathbf{k}_{1}$; comme ce vecteur jouera le rôle d'impulsion initiale relative de la collision, on peut dire que cette dernière définit dans tous les cas l'axe $\mathrm{O} z$. Passant des variables angulaires de $\hat{\mathbf{k}}_{2}$ aux composantes cartésiennes de ce vecteur unitaire, nous faisons apparaître l'exponentielle :

$$
\mathrm{e}^{-i k_{+} z^{\prime}} \mathrm{e}^{i k_{-}\left|\hat{k}_{2 x} x^{\prime}+k_{2 y} y^{\prime}+k_{2 z} z^{\prime}\right|} .
$$

Développant $\rho_{\mathrm{WL}}^{(+)}$comme en (41), nous ne gardons pour le moment que le terme d'ordre le plus bas ; cette opération introduit les mêmes fonctions delta qu'en (42a) - à un changement près de $k_{+}$en $k_{-}$et de $\hat{k}$ en $\hat{k}_{2}-$, ce qui impose :

$$
k_{-} \hat{k}_{2 z}= \pm k_{-} \text {. }
$$

La nappe correspondant au signe + de cette égalité introduit une intégrale sur $z^{\prime}$ analogue à (43), ce qui permet de montrer que :

$$
\hat{\mathbf{k}}_{2}=\hat{\mathbf{k}}_{1}
$$

(le jacobien vaut un) et d'utiliser (42d) (en y substituant $k_{-}$à $k_{+}, \hat{\mathbf{k}}_{2}$ à $\hat{\mathbf{k}}$ ) pour intégrer sur les deux composantes de $\hat{\mathbf{k}}_{2}$; pour finir :

$$
\frac{p}{\mu} \int \mathrm{d}^{2} \hat{k} \sigma_{p}(\hat{\mathbf{p}} \cdot \hat{\mathbf{k}}) \rho_{\mathrm{WL}}^{(+)}\left(\mathbf{R}=\mathbf{r}_{1}, \mathbf{r}=\mathbf{0}, \mathbf{P}, p \hat{\mathbf{k}}\right)
$$

où l'on retrouve la définition habituelle de la section efficace différentielle :

$$
\sigma_{p}(\hat{\mathbf{p}} \cdot \hat{\mathbf{k}})=\frac{16 \pi^{4} \mu^{2}}{\hbar^{4}}\left|T_{p / \hbar}(\hat{\mathbf{p}} \cdot \hat{\mathbf{k}})\right|^{2} .
$$

La nappe correspondant au signe moins dans (50) fait, comme pour le terme de diffusion vers l'avant, intervenir une fonction delta dont l'argument ne peut jamais s'annuler, de sorte que sa contribution est nulle.

\section{Effets de retard et de réfraction.}

Reprenons maintenant les calculs précédents en gardant les premiers termes négligés, soit dans la dépendance de $\rho$ en $\mathbf{R}$, soit en $\mathbf{r}$. Nous examinerons successivement les termes linéaires et quadratiques en $T$.

4.1 DifFusion VERS L'AVANT. - Nous partons à nouveau de la formule (34) - sommée sur $\mathbf{r}$ après la substitution (35) - et nous reprenons l'une après l'autre les approximations faites pour calculer cette intégrale.

4.1.1 Gradients par rapport à $\mathbf{R}$. - C'est le deuxième terme du second membre de (37) qui nous intéresse maintenant. Les sommations sur $x$ et $y$ (l'axe $\mathrm{O} z$ est à nouveau pris parallèle à p) donnent alors :

$$
-\frac{1}{2} \int \mathrm{d} x \int \mathrm{d} y x \mathrm{e}^{i\left(\kappa_{x} x+\kappa_{y} y\right)}=2 i \pi^{2} \delta^{\prime}\left(\kappa_{x}\right) \delta\left(\kappa_{y}\right)
$$


(et un terme semblable où $x$ et $y$ sont intervertis). Nous obtenons donc, en notant $X$ et $Y$ les composantes de $\mathbf{R}$ sur les axes $O x$ et $O y$ :

$$
\begin{aligned}
& -\left(p / 2 \hbar^{2}\right) \int \mathrm{d} \kappa \int \mathrm{d}^{2} \hat{k} \int \mathrm{d}^{3} r^{\prime} \times \\
& \times\left\{\frac{\partial}{\partial \kappa_{x}}\left[\mathrm{e}^{i\left(\mathbf{k}_{-}-k_{+} \hat{\mathbf{k}}\right) \cdot \mathbf{r}^{\prime}} k_{+} T\left(\mathbf{k}_{+}, k_{+} \hat{\mathbf{k}}\right) \frac{\partial}{\partial X} \rho_{\mathrm{WL}}^{(+)}\left(\mathbf{R}=\mathbf{r}_{1}, \mathbf{r}^{\prime}, \mathbf{P}, \hbar \frac{k_{+} \hat{\mathbf{k}}+\mathbf{k}_{-}}{2}\right)\right]+i d(x, y)\right\}
\end{aligned}
$$

où, dans l'intégrale, après dérivation de la fonction par rapport à $\kappa_{x}$, il faut donner des valeurs nulles à $\kappa_{x}$ et $\kappa_{y}$ (comme plus haut, $\kappa$ remplace $\left.\kappa_{z}\right) ;$ id $(x, y)$ désigne le terme où les dérivations sont faites par rapport à $\kappa_{y}$ et $Y$. Dérivons tour à tour les divers termes.

(i) Dérivée de l'exponentielle ; comme :

$$
k_{ \pm}=\left[\frac{p^{2}}{\hbar^{2}} \pm \frac{\mathbf{p} \cdot \kappa}{\hbar}+\frac{\kappa^{2}}{4}\right]^{1 / 2}
$$

il vient :

$$
\nabla_{\kappa} k_{ \pm}=\frac{1}{2 k_{ \pm}}\left[\frac{\kappa}{2} \pm \frac{\mathbf{p}}{\hbar}\right]
$$

On voit ainsi que, lorsque $\kappa_{x}$ et $\kappa_{y}$ sont nuls, les dérivées de $k_{ \pm}$en $\kappa_{x}$ et $\kappa_{y}$ sont nulles. Le seul terme à dériver dans l'exponentielle est donc celui en $\mathbf{k}_{-} \cdot \mathbf{r}^{\prime}$. On a :

$$
\left[\frac{\partial}{\partial \kappa_{x}} \mathrm{e}^{i \mathbf{k}_{-} \cdot \mathbf{r}^{\prime}}\right]_{\kappa_{x, y}=0}=-\frac{i x^{\prime}}{2} \mathrm{e}^{i k_{-} z^{\prime}}
$$

ce qui introduit la contribution à (54) :

$$
\begin{aligned}
\frac{i p}{4 \hbar^{2}} \int \mathrm{d} \kappa \int \mathrm{d}^{2} \hat{k} \int \mathrm{d}^{3} r^{\prime} \mathrm{e}^{i\left(\tilde{k}_{-} \hat{\mathbf{p}}-k_{+} \hat{\mathbf{k}}\right) \cdot \mathbf{r}^{\prime}} k_{+} T\left(\tilde{k}_{+} \hat{\mathbf{p}}, k_{+} \hat{\mathbf{k}}\right) \times \\
\times\left[x^{\prime} \frac{\partial}{\partial X}+y^{\prime} \frac{\partial}{\partial Y}\right] \rho_{\mathrm{WL}}^{(+)}\left(\mathbf{R}=\mathbf{r}_{1}, \mathbf{r}^{\prime}, \mathbf{P}, \hbar \frac{k_{+} \hat{\mathbf{k}}+\hat{k}_{-} \hat{\mathbf{p}}}{2}\right) .
\end{aligned}
$$

Nous pouvons alors, comme au paragraphe 3 , ignorer la dépendance en $\mathbf{r}^{\prime}$ de $\rho_{\text {WL }}^{(+)}$(elle introduirait des termes du second ordre en gradients), ce qui permet d'utiliser la relation :

$$
\int \mathrm{d} x^{\prime} \int \mathrm{d} y^{\prime} x^{\prime} \mathrm{e}^{i\left(\hat{k}_{-} \hat{\mathbf{p}}-k_{+} \hat{\mathbf{k}}\right) \cdot \mathbf{r}^{\prime}}=-4 i \pi^{2} \delta^{\prime}\left(k_{+} \hat{k}_{x}\right) \delta\left(k_{+} \hat{k}_{y}\right) \mathrm{e}^{i\left(\hat{k}_{-}-k_{+} k_{z}\right) z^{\prime}} .
$$

Le raisonnement est alors semblable à celui de la fin du paragraphe 3.2 ; on a deux déterminations du vecteur $k_{+} \hat{\mathbf{k}}$, comme en (42b), dont la première donne (43) pour l'intégrale sur $z^{\prime}$, ce qui permet de montrer que $\hat{\mathbf{k}}$ et $\hat{\mathbf{p}}$ sont égaux et de remplacer le second membre de (57a) par :

$$
-\frac{i}{k_{+}}\left[\frac{2 \pi}{k_{+}}\right]^{2} \delta^{\prime}\left(\hat{k}_{x}\right) \delta\left(\hat{k}_{y}\right) \mathrm{e}^{i\left(\hat{k}_{-}-k_{+} k_{z}\right) z^{\prime}}
$$


Le calcul de l'intégrale sur $\hat{\mathbf{k}}$ en coordonnées cartésiennes conduit donc à dériver la fonction sous l'intégrale de (56) par rapport à $\hat{k}_{x}$ (le jacobien étant stationnaire lorsque (42c) est vérifiée, il n'est pas nécessaire de le dériver); la fonction $T$ dépend de l'angle de collisión, plus précisément de :

$$
\cos \theta=\hat{\mathbf{p}} \cdot \hat{\mathbf{k}}
$$

qui est maximal et n'introduit pas de terme par dérivation; pour la même raison, l'exponentielle qui subsiste au second membre de (57) ne contribue pas; pour finir, la seule dépendance qui importe ici est celle de $\rho_{\mathrm{WL}}^{(+)}$elle-même, par l'intermédiaire de sa dépendance en impulsion. On obtient ainsi :

$$
\begin{aligned}
\left(\pi^{2} p / \hbar^{2}\right) \int \mathrm{d} \kappa \int \mathrm{d} z^{\prime} \mathrm{e}^{-i \kappa z^{\prime}}\left[k_{+}\right]^{-2} & T_{k_{+}}(1) \times \\
& \times \frac{\hbar k_{+}}{2}\left[\frac{\partial}{\partial p_{x}} \frac{\partial}{\partial X}+\frac{\partial}{\partial p_{y}} \frac{\partial}{\partial Y}\right] \rho_{\mathrm{WL}}^{(+)}\left(\mathbf{R}=\mathbf{r}_{1}, \mathbf{0}, \mathbf{P}, \mathbf{p}\right) .
\end{aligned}
$$

La sommation sur $z^{\prime}$ introduit à nouveau (43); regroupant le terme obtenu avec le terme complexe conjugué, issu du terme en $T^{*}$, nous obtenons ainsi :

$$
2 \pi^{3} \operatorname{Re}\left\{T_{p / \hbar}(1)\right\} \nabla_{\mathbf{p}} \cdot \nabla_{\mathbf{R}}^{+} \rho_{\mathrm{WL}}^{(+)}\left(\mathbf{R}=\mathbf{r}_{1}, \mathbf{r}=\mathbf{0}, \mathbf{P}, \mathbf{p}\right)
$$

avec la notation :

$$
\nabla_{\mathbf{p}} \cdot \nabla_{\mathbf{R}}^{\perp}=\frac{\partial}{\partial p_{x}} \frac{\partial}{\partial X}+\frac{\partial}{\partial p_{y}} \frac{\partial}{\partial Y}
$$

La nappe où $\hat{\mathbf{k}}$ et $\hat{\mathbf{p}}$ sont opposés est ignorée pour les mêmes raisons que précédemment (impossibilité d'annuler l'argument de la fonction delta).

(ii) Dérivée de $T\left(\mathbf{k}_{+}, k_{+} \hat{\mathbf{k}}\right)$; cet élément de matrice de l'opérateur $T$ dépend du module de l'impulsion relative, $k_{+}$, dont la dérivée est nulle d'après (55b), et du cosinus de l'angle de collision :

$$
\hat{\mathbf{k}}_{+} \cdot \hat{\mathbf{k}}=\frac{p}{\hbar k_{+}} \hat{k}_{z}+\frac{1}{2 k_{+}}\left(\kappa_{x} \hat{k}_{x}+\kappa_{y} \hat{k}_{y}+\kappa_{z} \hat{k}_{z}\right) .
$$

La dérivée en $\kappa_{x}$ introduit donc le facteur $+\hat{k}_{x} / 2$; comme il n'y a maintenant plus de $x^{\prime}$ ou de $y^{\prime}$ dans l'intégrale, il apparaît les mêmes fonctions delta qu'au paragraphe 3.2, qui annulent $\hat{k}_{x}$; le résultat est donc nul.

(iii) Dérivée de $\rho_{\mathrm{WL}}^{(+)}$; c'est à nouveau la dépendance de $\rho_{\mathrm{WL}}^{(+)}$en $\mathbf{p}$ qui intervient et donne lieu au terme :

$$
\begin{aligned}
\frac{p}{8 \hbar} \int \mathrm{d} \kappa \int \mathrm{d}^{2} \hat{k} \int & \mathrm{d}^{3} r^{\prime} \mathrm{e}^{i\left(\tilde{k}_{-} \mathbf{p}-k_{+} \hat{\mathbf{k}}\right) \cdot \mathbf{r}^{\prime}} \times \\
& \times k_{+} T\left(\tilde{k}_{+} \hat{\mathbf{p}}, k_{+} \hat{\mathbf{k}}\right) \nabla_{\mathbf{p}} \cdot \nabla_{\mathbf{R}}^{+} \rho_{\mathrm{WL}}^{(+)}\left(\mathbf{R}=\mathbf{r}_{1}, \mathbf{r}^{\prime}, \mathbf{P}, \hbar \frac{k_{+} \hat{\mathbf{k}}+\tilde{k}_{-} \hat{\mathbf{p}}}{2}\right) .
\end{aligned}
$$

Le calcul à effectuer est alors le même que pour la formule (38), avec maintenant une dérivée de $\rho_{\mathrm{WL}}^{(+)}$et un coefficient légèrement différent ; il donne une contribution égale à (60), qu'il convient donc de doubler. 
4.1.2 Gradients par rapport à $\mathbf{r}^{\prime}$. - Nous partons maintenant de (38) et nous examinons la contribution des termes du premier ordre en $\mathbf{r}^{\prime}$ dans le second membre de (41). Nous considérerons successivement les composantes transverses, puis longitudinale, de ce vecteur.

(i) Termes en $x^{\prime}$ et $y^{\prime}$; utilisant encore (57), nous obtenons :

$$
\begin{aligned}
-4\left(\pi^{2} p / \hbar^{2}\right) \int \mathrm{d} \kappa \int \mathrm{d} z^{\prime} \mathrm{e}^{-i \kappa z^{\prime}}\left[k_{+}\right]^{-2} \times & \\
\times & \left\{\frac{\partial}{\partial \hat{k}_{x}}\left[T_{k_{+}}(\hat{\mathbf{p}} \cdot \hat{\mathbf{k}}) \frac{\partial}{\partial x^{\prime}} \rho_{\mathrm{WL}^{+}}^{(+)}\left(\mathbf{R}=\mathbf{r}_{1}, \mathbf{0}, \mathbf{P}, \mathbf{p}\right)\right]+i d(x, y)\right\}
\end{aligned}
$$

(pour la première nappe); après dérivation par rapport à $\hat{k}_{x}$ ou $\hat{k}_{y}$, il faut annuler ces variables. Pour les mêmes raisons que plus haut, la seule fonction à dériver est $\rho_{\mathrm{WL}}^{(+)}$et l'on obtient, après avoir utilisé (43), et regroupé avec le terme complexe conjugué :

$$
-8 \pi^{3} \operatorname{Re}\left\{T_{p / \hbar}(1)\right\} \nabla_{\mathbf{p}} \cdot \nabla_{\mathbf{r}}^{\perp} \rho_{\mathrm{WL}}^{(+)}\left(\mathbf{R}=\mathbf{r}_{1}, \mathbf{r}=\mathbf{0}, \mathbf{P}, \mathbf{p}\right)
$$

(en introduisant une notation semblable à (61) pour la dérivée par rapport à r).

(ii) Terme en $z^{\prime}$; nous repartons encore de (38), mais cette fois en prenant le terme en $z^{\prime}$ dans le second membre de (41). Nous pouvons donc toujours nous servir des égalités (42a) et (42b) mais, plutôt que (43), il faut maintenant utiliser la relation :

$$
\int \mathrm{e}^{i\left(k_{+}-\tilde{k}_{-}\right) z^{\prime}} z^{\prime} \mathrm{d} z^{\prime}=-2 i \pi \delta^{\prime}(\kappa)
$$

qui se démontre de façon analogue. Comme, pour la première nappe, la transformée entrante ne dépend pas de $\kappa$, la seule fonction à dériver est :

$$
\frac{1}{k_{+}}\left[T_{k_{+}}(1)\right]
$$

et le résultat obtenu pour finir est, après regroupement avec le terme complexe conjugué :

$$
-\frac{8 \pi^{3} p}{\hbar^{2}} \frac{\mathrm{d}}{\mathrm{d} k}\left[\frac{T_{k}(1)}{k}\right]_{k=p / \hbar} \hat{\mathbf{p}} \cdot \nabla_{\mathbf{r}} \rho_{\mathrm{WL}}^{(+)}\left(\mathbf{R}=\mathbf{r}_{1}, \mathbf{r}=\mathbf{0}, \mathbf{P}, \mathbf{p}\right) .
$$

4.2 Diffusion latérale. - C'est maintenant de la formule (47) que nous repartons pour examiner les corrections laissées de côté au paragraphe 3.

4.2.1 Dérivée en $\mathbf{R}$ de $\rho$. - Le terme du premier ordre en $\mathbf{r}$ au second membre de (37) introduit, soit $x$, soit $y$ sous l'intégrale (le terme en $z$ disparaît à cause de la fonction delta). Nous utilisons donc à nouveau la formule (53), et nous obtenons :

$$
\begin{aligned}
-\left(i \pi p \mu \hbar^{-4}\right) \int \mathrm{d} \kappa \int \mathrm{d}^{2} \hat{k}_{1} \int \mathrm{d}^{2} \hat{k}_{2} \int \mathrm{d}^{3} r^{\prime} \times \\
\times\left\{\frac { \partial } { \partial \kappa _ { x } } \left[\mathrm{e}^{i\left(k_{-} \hat{\mathbf{k}}_{2}-k_{+} \hat{\mathbf{k}}_{1}\right) \cdot \mathbf{r}^{\prime}} k_{+} k_{-} T\left(\mathbf{k}_{+}, k_{+} \hat{\mathbf{k}}_{1}\right) T^{*}\left(\mathbf{k}_{-}, k_{-} \hat{\mathbf{k}}_{2}\right)\right.\right. \\
\left.\times \frac{\partial}{\partial X} \rho_{\mathrm{WL}}^{(+)}\left(\mathbf{R}=\mathbf{r}_{1}, \mathbf{r}^{\prime}, \mathbf{P}, \hbar \frac{k_{+} \hat{\mathbf{k}}_{1}+k_{-} \hat{\mathbf{k}}_{2}}{2}\right)+i d .(x, y)\right\}
\end{aligned}
$$


qui est l'analogue de (54) pour la diffusion latérale. Comme pour cette égalité, il faut annuler les composantes transverses de $\kappa$ après dérivation, ce qui élimine toute contribution des dérivées de $k_{+}$et $k_{-}$; ni l'exponentielle ni $\rho$ n'ont donc ici à être dérivées, mais seulement $T$ et sa complexe conjuguée. On a :

$$
T\left(\mathbf{k}_{+}, k_{+} \hat{\mathbf{k}}_{1}\right)=T_{k_{+}}\left(\hat{\mathbf{k}}_{+} \cdot \hat{\mathbf{k}}_{1}\right)
$$

et donc :

$$
\left[\frac{\partial}{\partial \kappa_{x}} T\left(\mathbf{k}_{+}, k_{+} \hat{\mathbf{k}}_{1}\right)\right]_{\kappa_{x}=\kappa_{y}=0}=\frac{1}{2 k_{+}} \hat{k}_{1 x} T_{k_{+}}^{\prime}\left(\hat{\mathbf{k}}_{+} \cdot \hat{\mathbf{k}}_{1}\right)
$$

où $T^{\prime}$ désigne la dérivée de $T$ par rapport au cosinus de l'angle de collision :

$$
T_{k}^{\prime}(\cos \theta)=\frac{\partial}{\partial \cos \theta} T_{k}(\cos \theta)
$$

On peut donc écrire (69) sous la forme :

$$
\begin{aligned}
& -\left(i \pi p \mu \hbar^{-4}\right) \int \mathrm{d} \kappa \int \mathrm{d}^{2} \hat{k}_{1} \int \mathrm{d}^{2} \hat{k}_{2} \int \mathrm{d}^{3} r^{\prime}\left[k_{+} k_{-}\right] \mathrm{e}^{i\left(k_{-} \hat{\mathbf{k}}_{2}-k_{+} \hat{\mathbf{k}}_{1}\right) \cdot \mathbf{r}^{\prime}} \times \\
& \quad \times\left[\left(T_{k_{+}}^{\prime} T_{k_{-}}^{*}\right) \frac{\hat{\mathbf{k}}_{1}}{2 k_{+}}-\left(T_{k_{+}} T_{k_{-}}^{\prime *}\right) \frac{\hat{\mathbf{k}}_{2}}{2 k_{-}}\right] \cdot \nabla_{\mathbf{R}}^{+} \rho_{\mathrm{WL}}^{(+)}\left(\mathbf{R}=\mathbf{r}_{1}, \mathbf{r}^{\prime}, \mathbf{P}, \hbar \frac{k_{+} \hat{\mathbf{k}}_{1}+k_{-} \hat{\mathbf{k}}_{2}}{2}\right)
\end{aligned}
$$

où le cosinus de l'angle de collision vaut $\hat{\mathbf{k}}_{+} \cdot \hat{\mathbf{k}}_{1}$ pour $T, \hat{\mathbf{k}}_{-} \cdot \hat{\mathbf{k}}_{2}$ pour $T^{*}$. Les deux termes dans le crochet sont complexes conjugués. L'égalité (71) est du même type que (48), et la suite du calcul est identique; on trouve ainsi :

$$
4 \pi^{4} \mu \hbar^{-3} \int \mathrm{d}^{2} \hat{k}\left[i T_{k}(\hat{\mathbf{p}} \cdot \hat{\mathbf{k}}) T_{k}^{\prime *}(\hat{\mathbf{p}} \cdot \hat{\mathbf{k}})+\text { c.c. }\right] \hat{\mathbf{k}} \cdot \nabla_{\mathbf{R}}^{\perp} \rho_{\mathrm{WL}}^{(+)}\left(\mathbf{R}=\mathbf{r}_{1}, \mathbf{r}=\mathbf{0}, \mathbf{P}, p \hat{\mathbf{k}}\right)
$$

où le symbole $\perp$ rappelle que le gradient de $\rho$ considéré n'a que des composantes perpendiculaires à p.

4.2.2 Dérivée en $x^{\prime}$ et $y^{\prime}$ de $\rho$. - Partant maintenant de (48), nous examinons la contribution des termes en $x^{\prime}$ et $y^{\prime}$ dans le second membre de (41) (l'axe $\mathrm{O} z$ est parallèle à $\hat{\mathbf{k}}_{1}$ ). Il apparaît alors à nouveau l'exponentielle (49), multipliée par $x^{\prime}$ ou $y^{\prime}$, ce qui par intégration sur ces variables donne maintenant (pour la première nappe) :

$$
-4 i \pi^{2}\left[k_{-}\right]^{-3} \mathrm{e}^{-i k_{+} z^{\prime}} \mathrm{e}^{i k_{-} k_{2 z} z^{\prime}} \delta^{\prime}\left(\hat{k}_{2 x}\right) \delta\left(\hat{k}_{2 y}\right)
$$

(ou la formule symétrique en $x$ et $y$ ). Deux sortes de dérivées sont ainsi introduites.

(i) Dérivée de $T$; le terme correspondant donne :

$$
\begin{aligned}
& \left(8 i \pi^{3} p \mu \hbar^{-4}\right) \int \mathrm{d} \kappa \int \mathrm{d}^{2} \hat{k} \int \mathrm{d} z^{\prime} k_{+}\left[k_{-}\right]^{-2} \times \\
& \times T_{k_{+}}\left(\hat{\mathbf{k}}_{+} \cdot \hat{\mathbf{k}}\right) T_{k_{-}^{*}}^{*}\left(\hat{\mathbf{k}}_{-} \cdot \hat{\mathbf{k}}\right)\left[\hat{p}_{x} \frac{\partial}{\partial x}+\hat{p}_{y} \frac{\partial}{\partial y}\right] \rho_{\mathrm{WL}}^{(+)}(\cdots)
\end{aligned}
$$


soit en utilisant (43) comme plus haut :

$$
16 \pi^{4} \mu \hbar^{-3} \int \mathrm{d}^{2} \hat{k}\left[i T_{p}(\hat{\mathbf{p}} \cdot \hat{\mathbf{k}}) T_{p}^{\prime *}(\hat{\mathbf{p}} \cdot \hat{\mathbf{k}})\right]\left[\hat{p}_{x} \frac{\partial}{\partial x}+\hat{p}_{y} \frac{\partial}{\partial y}\right] \rho_{\mathrm{WL}}^{(+)}(\cdots)
$$

Mais nous aurions pu raisonner en intervertissant les rôles des deux vecteurs $\mathbf{k}_{1}$ et $\mathbf{k}_{2}$; nous aurions ainsi obtenu l'expression complexe conjuguée. Par demi-somme, on remplace donc $(74 b)$ par :

$$
\begin{aligned}
8 \pi^{4} \mu \hbar^{-3} \int \mathrm{d}^{2} \hat{k}\left[-i T_{p / \hbar}^{\prime}(\hat{\mathbf{p}} \cdot \hat{\mathbf{k}}) T_{p / \hbar}^{*}(\hat{\mathbf{p}} \cdot \hat{\mathbf{k}})+\text { c.c. }\right] \times \\
\left.\quad \times[\hat{\mathbf{p}}-(\hat{\mathbf{p}} \cdot \hat{\mathbf{k}}) \hat{\mathbf{k}}] \cdot \nabla_{\mathbf{r}} \rho_{W_{L}^{(+)}}^{(\mathbf{R}}=\mathbf{r}_{1}, \mathbf{r}=\mathbf{0}, \mathbf{P}, p \hat{\mathbf{k}}\right) .
\end{aligned}
$$

(ii) Dérivée de $\rho$; un calcul du même type conduit à :

$$
8 i \pi^{4} p \mu \hbar^{-3} \int \mathrm{d}^{2} \hat{k}\left|T_{p / \hbar}(\hat{\mathbf{p}} \cdot \hat{\mathbf{k}})\right|^{2}\left[\frac{\partial}{\partial p_{x}} \frac{\partial}{\partial x}+\frac{\partial}{\partial p_{y}} \frac{\partial}{\partial y}\right] \rho_{\mathrm{WL}}^{(+)}(\cdots)
$$

qui est imaginaire pur ; renversant le rôle de $\mathbf{k}_{1}$ et $\mathbf{k}_{2}$, nous aurions obtenu le terme opposé. Il n'y a donc pas de contribution de cette dérivée double de $\rho$.

4.2.3 Dérivée en $z^{\prime}$ de $\rho$. - Intégrée sur $x^{\prime}$ et $y^{\prime}$, l'exponentielle (49) redonne maintenant les fonctions delta de (42a) qui imposent (50) dont nous ne retenons comme plus haut que la première nappe ; la sommation sur $z^{\prime}$ introduit pour sa part la relation (66), dont la fonction $\delta^{\prime}$ conduit à effectuer les deux dérivées en $\kappa$ :

$$
\frac{\mathrm{d}}{\mathrm{d} \kappa}\left[\frac{k_{+}}{k_{-}}\right]=\frac{p}{\hbar k_{-}^{2}}
$$

et :

$$
\frac{\mathrm{d}}{\mathrm{d} \kappa}\left[T_{k_{+}}\left(\hat{\mathbf{k}}_{+} \cdot \hat{\mathbf{k}}\right) T_{k_{-}}^{*}\left(\hat{\mathbf{k}}_{-} \cdot \hat{\mathbf{k}}\right)\right]_{\kappa=0}=\frac{1}{2}\left[T_{p / \hbar}^{*}(\hat{\mathbf{p}} \cdot \hat{\mathbf{k}}) \frac{\mathrm{d}}{\mathrm{d} k} T_{k}(\hat{\mathbf{p}} \cdot \hat{\mathbf{k}})-\mathrm{c.c} .\right]
$$

(où la dérivée en $k$ de $T$ est prise en $k=p / \hbar$, comme en 68). Il vient ainsi :

$$
\begin{aligned}
-8 i \pi^{4} p \mu \hbar^{-4} \int \mathrm{d}^{2} \hat{k}\left\{\frac{2 \hbar}{p}\left|T_{p / \hbar}(\hat{\mathbf{p}} \cdot \hat{\mathbf{k}})\right|^{2}+\left[T_{p / \hbar}^{*}(\hat{\mathbf{p}} \cdot \hat{\mathbf{k}}) \frac{\mathrm{d}}{\mathrm{d} k} T_{k}(\hat{\mathbf{p}} \cdot \hat{\mathbf{k}})-\text { c.c. }\right]\right\} \times \\
\times \hat{\mathbf{k}} \cdot \nabla_{\mathrm{r}} \rho_{\mathrm{WL}}^{(+)}\left(\mathbf{R}=\mathbf{r}_{1}, \mathbf{r}=\mathbf{0}, \mathbf{P}, p \hat{\mathbf{k}}\right) .
\end{aligned}
$$

Mais nous aurions ici aussi pu obtenir l'expression complexe conjuguée en intervertissant les rôles de $\mathbf{k}_{1}$ et $\mathbf{k}_{2}$. Par demi-somme, il reste donc :

$$
8 \pi^{4} p \mu \hbar^{-4} \int \mathrm{d}^{2} \hat{k}\left[i T_{p / \hbar}(\hat{\mathbf{p}} \cdot \hat{\mathbf{k}}) \frac{\mathrm{d}}{\mathrm{d} k} T_{k}^{*}(\hat{\mathbf{p}} \cdot \hat{\mathbf{k}})+\text { c.c. }\right] \hat{\mathbf{k}} \cdot \nabla_{\mathbf{r}} \rho_{W L}^{(+)}\left(\mathbf{R}=\mathbf{r}_{1}, \mathbf{r}=\mathbf{0}, \mathbf{P}, p \hat{\mathbf{k}}\right) .
$$

\section{Récapitulation.}

Regroupant les résultats des paragraphes 3 et 4 , nous effectuons la somme de (45) et (51) pour les termes d'ordre zéro en gradients, de (6) [doublé], (65), (68), ainsi que de (72), (75) et (80), et nous obtenons : 


$$
\begin{aligned}
\int \mathrm{d}^{3} r_{2}\left[\frac{\partial}{\partial t}+\sum_{i=1}^{2} \frac{\mathbf{p}_{i}}{m} \cdot \nabla_{\mathbf{r}_{i}}\right] \rho_{\mathrm{W}}^{\mathrm{L}} & =I_{\text {Boltz. }}\left[\rho_{\mathrm{WL}}^{(+)}\right]+ \\
& +\frac{p}{\mu}\left\{-2 \xi_{F}(k) \hat{\mathbf{p}} \cdot \nabla_{\mathbf{r}} \rho_{\mathrm{WL}}^{(+)}+\frac{\Delta_{F}(k)}{2}\left[\nabla_{\mathbf{R}}^{\perp}-2 \nabla_{\mathbf{r}}^{\perp}\right] \cdot \nabla_{\mathbf{p}} \rho_{\mathrm{WL}}^{(+)}+\right. \\
& +\int \mathrm{d}^{2} \hat{k}^{\prime}\left[2 \xi_{k} \hat{\mathbf{k}}^{\prime} \cdot \nabla_{\mathbf{r}} \rho_{\mathrm{WL}}^{(+)}+\Delta_{k}(\theta) \times\right. \\
& \left.\left.\times\left[\left(\hat{\mathbf{k}}^{\prime}-\left(\hat{\mathbf{k}}^{\prime} \cdot \hat{\mathbf{p}}\right) \hat{\mathbf{p}}\right) \cdot \nabla_{\mathbf{R}}+2\left(\hat{\mathbf{p}}-\left(\hat{\mathbf{k}}^{\prime} \cdot \hat{\mathbf{p}}\right) \hat{\mathbf{k}}^{\prime}\right) \cdot \nabla_{\mathbf{r}}\right] \rho_{\mathrm{WL}}^{(+)}\right]\right\}
\end{aligned}
$$

où $I_{\text {Boltz. }}$ est le terme de collision habituel de Boltzmann (avant sommation sur l'impulsion $\mathbf{p}_{2}$ du partenaire de collision), et :

$$
\begin{aligned}
& \xi_{F}(k)=\frac{4 \pi^{3} \mu}{\hbar^{2}} \frac{\mathrm{d}}{\mathrm{d} k} \operatorname{Re}\left\{\frac{T_{k}(1)}{k}\right\} \\
& \Delta_{F}(k)=\frac{8 \pi^{3} \mu}{p} \operatorname{Re}\left\{T_{k}(1)\right\} \\
& \xi_{k}(\theta)=\frac{4 \pi^{4} \mu^{2}}{\hbar^{4}}\left[i T_{k}(\cos \theta) \frac{\mathrm{d}}{\mathrm{d} k} T_{k}^{*}(\cos \theta)+\text { c.c. }\right] \\
& \Delta_{k}(\theta)=\frac{4 \pi^{4} \mu^{2}}{\hbar^{3} p}\left[i T_{k}(\cos \theta) T_{k}^{* *}(\cos \theta)+\text { c.c. }\right] .
\end{aligned}
$$

Ces quantités jouent un peu le rôle de sections efficaces généralisées, bien qu'elles ne soient pas nécessairement positives (les sections efficaces elles-mêmes ont déjà été définies en (46) et (52)) ; à part la seconde, qui est homogène à une section efficace multipliée par $\hbar$, les trois autres sont homogènes à une section efficace multipliée par une longueur. On a dans (81) remplacé la variable muette $\hat{\mathbf{k}}$ par $\hat{\mathbf{k}}^{\prime}$ et noté :

$$
\mathbf{p}=\hbar \mathbf{k} \quad \cos \theta=\hat{\mathbf{p}} \cdot \hat{\mathbf{k}}^{\prime}
$$

où $\theta$ est l'angle de collision; les variables de position du centre de masse ou de la particule relative, ainsi que celles d'impulsion, sont reliées aux positions et impulsions des particules par les égalités habituelles (12a). Rappelons enfin que le signe $\perp$ symbolise la projection sur le plan perpendiculaire à $\mathbf{p}$; par exemple :

$$
\nabla_{\mathbf{R}}^{\perp} \cdot \nabla_{\mathbf{p}}=\nabla_{\mathbf{R}} \cdot \nabla_{\mathbf{p}}-\left(\hat{\mathbf{p}} \cdot \nabla_{\mathbf{R}}\right)\left(\hat{\mathbf{p}} \cdot \nabla_{\mathbf{p}}\right) .
$$

L'égalité (81) est générale, sa seule hypothèse de validité étant que les variations spatiales de la transformée de Wigner avant collision ne soient pas trop rapides.

Un cas particulier important est celui où les deux particules ne sont pas initialement corrélées, ce qui permet de définir la transformée libre entrante par l'intermédiaire de deux fonctions séparées $f_{1}$ et $f_{2}$ et d'écrire :

$$
\rho_{\mathrm{WL}}^{(+)}(\mathbf{R}, \mathbf{r}, \mathbf{P}, \mathbf{p})=f_{1}\left(\mathbf{r}_{1}, \mathbf{p}_{1}\right) \times f_{2}\left(\mathbf{r}_{2}, \mathbf{p}_{2}\right) .
$$

Dans cette relation, les variables du premier et du second membre sont reliées par (12a). Alors (81) devient (nous considérons maintenant que les $\xi$ et les $\Delta$, comme les sections efficaces, sont des fonctions de l'impulsion relative $p$, sans introduire le coefficient $\hbar$ correspondant pour simplifier) : 


$$
\begin{aligned}
\int \mathrm{d}^{3} r_{2}\left[\frac{\partial}{\partial t}+\frac{\mathbf{p}_{1}}{m} \cdot \nabla_{\mathbf{r}_{1}}\right] \rho_{\mathrm{W}}^{\mathrm{L}}\left(\mathbf{r}_{1}, \mathbf{r}_{2}\right. & \left., \mathbf{p}_{1}, \mathbf{p}_{2} ; t\right)=\frac{p}{\mu}\left\{-\sigma_{T}(p) f_{1}(1) f_{2}(2)+\right. \\
& +\int \mathrm{d}^{2} \hat{k} \sigma_{p}(\theta) f_{1}\left(1^{\prime}\right) f_{2}\left(2^{\prime}\right) \\
& -\xi_{F}(p) \hat{\mathbf{p}} \cdot\left[f_{2}(2) \nabla f_{1}(1)-f_{1}(1) \nabla f_{2}(2)\right] \\
& +\Delta_{F}(p)\left[\nabla_{\mathbf{p}_{1}}-\nabla_{\mathbf{p}_{2}}\right] \cdot[f(1)(\nabla-\hat{\mathbf{p}}(\hat{\mathbf{p}} \cdot \nabla)) f(2)] \\
& +\int \mathrm{d}^{2} \hat{k}^{\prime} \xi_{p}(\theta) \hat{\mathbf{k}}^{\prime} \cdot\left[f_{2}\left(2^{\prime}\right) \nabla f_{1}\left(1^{\prime}\right)-f_{1}\left(1^{\prime}\right) \nabla f_{2}\left(2^{\prime}\right)\right] \\
& +\int \mathrm{d}^{2} \hat{k}^{\prime}\left(1-\hat{\mathbf{k}}^{\prime} \cdot \hat{\mathbf{p}}\right) \Delta_{p}(\theta) f_{2}\left(2^{\prime}\right)\left(\hat{\mathbf{k}}^{\prime}+\hat{\mathbf{p}}\right) \cdot \nabla f_{1}\left(1^{\prime}\right) \\
& \left.+\int \mathrm{d}^{2} \hat{k}^{\prime}\left(1+\hat{\mathbf{k}}^{\prime} \cdot \hat{\mathbf{p}}\right) \Delta_{p}(\theta) f_{1}\left(1^{\prime}\right)\left(\hat{\mathbf{k}}^{\prime}-\hat{\mathbf{p}}\right) \cdot \nabla f_{2}\left(2^{\prime}\right)\right\}
\end{aligned}
$$

Dans cette formule, les gradients pour lesquels la variable de dérivation n'est pas précisée sont pris par rapport à la variable de position; les variables de chacune des particules sont résumées sous la forme :

$$
(1),(2) \Leftrightarrow\left(\mathbf{r}_{1}, \mathbf{p}_{1,2}\right)
$$

(pour le terme de diffusion pour l'avant) et :

$$
\left(1^{\prime}\right),\left(2^{\prime}\right) \Leftrightarrow\left(\mathbf{r}_{1} ; \frac{\mathbf{p}_{1}+\mathbf{p}_{2}}{2} \pm \frac{\left|\mathbf{p}_{1}-\mathbf{p}_{2}\right|}{2} \hat{\mathbf{k}}^{\prime}\right)
$$

(pour le terme de diffusion latérale); les impulsions diffèrent, mais une seule position, $\mathbf{r}_{1}$, intervient.

Sous la forme (81) ou (86), la structure du terme de collision est la même. Les termes en $\xi$ introduisent des dérivées spatiales dont l'effet est, au premier ordre, celui d'une translation dans la direction de l'impulsion relative avant collision; ce sont des termes de retard (ou d'avance, suivant le signe de $\xi$ ) dans la collision. Les termes en $\Delta_{F}$ contiennent des dérivées à la fois en $\mathbf{r}$ et $\mathbf{p}$; un gradient de densité de la particule 2 produit un changement d'impulsion de la particule 1, suivant l'effet de réfraction discuté dans l'introduction; un terme en double dérivée par rapport aux position et impulsion de la particule 2 est également présent. L'analogue de ces effets pour la diffusion latérale est contenu dans les termes en $\Delta_{p}(\theta)$; il ne contient cependant que des dérivées spatiales, tout en n'étant pas un simple terme de retard pur puisqu'il ne fait pas intervenir une dérivée en $k$ (comme ce serait le cas pour un terme en $\xi$ ).

\section{Conclusion.}

Le formalisme que nous avons utilisé permet d'obtenir, en plus du terme de collision de l'équation de Boltzmann, des corrections du premier ordre en gradients qui incluent des effets « non locaux " de retard et de réfraction; la structure relativement complexe de ces termes justifie la méthode systématique employée pour les obtenir, car il aurait probablement été délicat de les obtenir de façon purement intuitive. Les corrections d'ordre supérieur pourraient être calculées par la même méthode, au prix de calculs plus développés, un peu comme la formule de Groenewold permet un développement à tous les ordres en gradients. Si 
de tels calculs s'avéraient nécessaires, il serait probablement plus avisé de revenir à la formule exacte (22) qui permet, elle aussi, l'écriture d'une équation cinétique, le prix à payer étant que le lien avec le formalisme de Boltzmann serait moins transparent.

Tous les calculs qui précèdent sont limités à l'étude d'une collision binaire. Cette collision est cependant traitée de façon rigoureuse, sans approximation semi-classique du type Thomas Fermi par exemple, puisque les résultats s'expriment en fonction de la matrice $S$, c'est-à-dire des déphasages quantiques exacts ; nous n'avons à aucun stade développé par rapport aux gradients du potentiel d'interaction, qui serait supposé varier lentement à l'échelle de la longueur d'onde de De Broglie, ou supposé que des variations rapides de $\rho$ ne se produisent pas pendant la collision. Si l'on préfère, la théorie restera valable tant que les rapports $a_{0} / L$ et $\lambda / L$ restent petits $\left(a_{0}\right.$ est la portée du potentiel, $\lambda$ la longueur d'onde de De Broglie et $L$ une grandeur macroscopique caractérisant les variations spatiales des états avant collision), mais on ne suppose rien du rapport $\lambda / a_{0}$.

Dans l'article B suivant, nous partirons des résultats ainsi obtenus pour écrire une équation cinétique pour un gaz quantique dilué ; ceci nous permettra en particulier de discuter le lien entre les termes du premier ordre en gradients et les effets de « champ moléculaire », bien connus dans les théories du type Hartree-Fock ou Landau.

\section{Remerciements.}

Merci à Pierre-Jean Nacher et Geneviève Tastevin qui ont pris la peine de lire cet article en détail et y ont suggéré bien des améliorations ; le fait qu'ils aient accepté de travailler sur les prolongements de ce travail et d'y apporter des idées nouvelles est un apport précieux. Merci également à Edouard Brézin pour d'éclairantes discussions et plusieurs suggestions fort utiles.

\section{Appendice A}

\section{Transformée de Wigner, quelques formules utiles.}

Soit $\rho$ l'opérateur densité d'une particule sans spin. La distribution de Wigner $\rho_{\mathrm{W}}$ est définie par ([11-14]) :

$$
\begin{aligned}
\rho_{\mathrm{W}}(\mathbf{r}, \mathbf{p}) & =(2 \pi \hbar)^{-3} \int \mathrm{d}^{3} x \mathrm{e}^{i \mathbf{x} \cdot \mathbf{p} / \hbar}\left\langle\mathbf{r}-\frac{\mathbf{x}}{2}|\rho| \mathbf{r}+\frac{\mathbf{x}}{2}\right\rangle \\
& =(2 \pi \hbar)^{-3} \int \mathrm{d}^{3} p^{\prime} \mathrm{e}^{i \mathbf{p}^{\prime} \cdot \mathbf{r} / \hbar}\left\langle\mathbf{p}+\frac{\mathbf{p}^{\prime}}{2}|\rho| \mathbf{p}-\frac{\mathbf{p}^{\prime}}{2}\right\rangle \\
& =(2 \pi \hbar)^{-3} \int \mathrm{d}^{3} k^{\prime} \mathrm{e}^{i \mathbf{k}^{\prime} \cdot \mathbf{r}}\left\langle\frac{\mathbf{p}}{\hbar}+\frac{\mathbf{k}^{\prime}}{2}|\rho| \frac{\mathbf{p}}{\hbar}-\frac{\mathbf{k}^{\prime}}{2}\right\rangle .
\end{aligned}
$$

Dans ces formules, les kets et bras sont normés en fonction des variables qui les repèrent, de sorte que :

$$
|\mathbf{p}\rangle=\hbar^{-3 / 2}|\mathbf{k}\rangle \text { si } \mathbf{p}=\hbar \mathbf{k} .
$$

Les coefficients sont choisis de façon que la condition :

$$
\operatorname{Tr}\{\rho\}=1
$$

soit équivalente à :

$$
\int d^{3} r \int d^{3} p \rho_{W}(\mathbf{r}, \mathbf{p})=1
$$


Pour une observable $\mathrm{A}$, sa transformée de Wigner sera définie sans les facteurs $(2 \pi \hbar)^{-3}$; de cette façon, une fonction de l'un des opérateurs $\mathbf{R}$ ou $\mathbf{P}$ reste par transformation de Wigner la même fonction de $\mathbf{r}$ ou $\mathbf{p}$.

Inversement, on a :

$$
\left\langle\mathbf{r}|\rho| \mathbf{r}^{\prime}\right\rangle=\hbar^{3} \int \mathrm{d}^{3} k \mathrm{e}^{i \mathbf{k} \cdot\left(\boldsymbol{r}-\mathbf{r}^{\prime}\right)} \rho_{\mathrm{W}}\left(\frac{\mathbf{r}+\mathbf{r}^{\prime}}{2}, \hbar \mathbf{k}\right)
$$

et :

$$
\left\langle\mathbf{p}|\rho| \mathbf{p}^{\prime}\right\rangle=\hbar^{-3}\left\langle\mathbf{k}|\rho| \mathbf{k}^{\prime}\right\rangle=\int \mathrm{d}^{3} r \mathrm{e}^{i\left(\mathbf{p}^{\prime}-\mathbf{p}\right) \cdot \mathbf{r} / \hbar} \rho_{\mathrm{W}}\left(\mathbf{r}, \frac{\mathbf{p}+\mathbf{p}^{\prime}}{2}\right)
$$

(pour une observable A, il faut multiplier les seconds membres par $2 \pi \hbar^{-3}$ ).

La transformée de Wigner d'un produit d'opérateurs n'est pas simplement le produit des transformées de Wigner; un développement en puissances de $\hbar$ (formule de Groenewold [15]) donne, au-delà de ce produit, toute une série de termes dont le premier est proportionnel au crochet de Poisson des deux transformées.

Une opération qui apparaît souvent dans notre travail est une transformation unitaire sur $\rho$ et le calcul de son effet sur la transformée de Wigner. Un calcul simple montre que, si la transformation est définie par un opérateur unitaire $U$ d'éléments de matrice $U\left(\mathbf{k}^{\prime}, \mathbf{k}\right)$, son action unitaire :

$$
\tilde{\boldsymbol{\rho}}=U \rho U^{\dagger}
$$

se traduit sur la transformée de Wigner par:

$$
\begin{aligned}
\tilde{\rho}_{\mathrm{W}}(\mathbf{r}, \mathbf{p})=(2 \pi)^{-3} \int \mathrm{d}^{3} \kappa \int \mathrm{d}^{3} k_{1} \int \mathrm{d}^{3} k_{2} \int & \mathrm{d}^{3} r^{\prime} \mathrm{e}^{i \kappa \cdot \mathbf{r}} \mathrm{e}^{i\left(\mathbf{k}_{2}-\mathbf{k}_{1}\right) \cdot \mathbf{r}^{\prime}} \times \\
& \times U\left(\mathbf{k}_{+}, \mathbf{k}_{1}\right) U^{*}\left(\mathbf{k}_{-}, \mathbf{k}_{2}\right) \rho_{\mathrm{W}}\left(\mathbf{r}^{\prime}, \hbar \frac{\mathbf{k}_{1}+\mathbf{k}_{2}}{2}\right)
\end{aligned}
$$

avec :

$$
\mathbf{k}_{ \pm}=\frac{\mathbf{p}}{\hbar} \pm \frac{\kappa}{2}
$$

Cette égalité a une structure générale que l'on rencontre souvent dans les calculs d'évolution de la transformée de Wigner ; elle contient plusieurs éléments qui apparaissent souvent dans les équations de cet article - cf. par exemple le second membre de (22).

Déterminons par exemple l'évolution de la transformée de Wigner d'un système comprenant deux particules en train de subir une collision. Nous reprenons donc les calculs des paragraphes 2 et suivants, mais cette fois pour la véritable transformée de Wigner. Utilisant la première des formules (A1), et insérant de chaque côté de l'opérateur densité des relations de fermeture sur la base des états stationnaires de collision, nous pouvons écrire :

$$
\begin{aligned}
\rho_{\mathrm{W}}(\mathbf{R}, \mathbf{r}, \mathbf{P}, \mathbf{p} ; t)=(2 \pi \hbar)^{-6} \int \mathrm{d}^{3} \kappa \int \mathrm{d}^{3} K \int \mathrm{d}^{3} k_{1} \int \mathrm{d}^{3} k_{2} \mathrm{e}^{i \kappa \cdot \mathbf{r}} \mathrm{e}^{i \mathbf{K} \cdot \mathbf{R}} \times \\
\times\left\langle\mathbf{k}_{+} \mid \Psi_{\mathbf{k}_{1}}^{(+)}\right\rangle\left\langle\Psi_{\mathbf{k}_{2}}^{(+)} \mid \mathbf{k}_{-}\right\rangle\left\langle\Psi_{\mathbf{k}_{1}}^{(+)}, \mathbf{K}_{+}|\rho(t)| \Psi_{\mathbf{k}_{2}}^{(+)}, \mathbf{K}_{-}\right\rangle
\end{aligned}
$$

(nous conservons les notations définies en (12)). L'opérateur densité étant placé entre des états propres de l'énergie, dont les différences des fréquences de Bohr sont données par:

$$
\omega_{1}-\omega_{2}=\hbar\left(k_{1}^{2}-k_{2}^{2}\right) / 2 \mu
$$


et par (15b), nous pouvons remplacer son élément de matrice par :

$$
\mathrm{e}^{i\left(\omega_{1}-\omega_{2}\right) t} \mathrm{e}^{-i \mathbf{P} \cdot \mathbf{K} t / M}\left\langle\Psi_{\mathbf{k}_{1}}^{(+)}, \mathbf{K}_{+}|\rho(0)| \Psi_{\mathbf{k}_{2}}^{(+)}, \mathbf{K}_{-}\right\rangle
$$

où $t=0$ est un instant initial. Si cet instant est choisi longtemps avant que commence la collision, on peut remplacer dans (A12) les états stationnaires de diffusion par des ondes planes, ce qui permet d'utiliser la relation d'inversion (A6) et donne :

$$
\begin{aligned}
\hbar^{6} \mathrm{e}^{i\left(\omega_{1}-\omega_{2}\right) t} \mathrm{e}^{-i \mathbf{P} \cdot \mathbf{K} t / M} \int \mathrm{d}^{3} r^{\prime} \int \mathrm{d}^{3} R^{\prime} \mathrm{e}^{i\left(\mathbf{k}_{2}-\mathbf{k}_{1}\right) \cdot \mathbf{r}^{\prime}} & \mathrm{e}^{-i \mathbf{K} \cdot \mathbf{R}^{\prime}} \times \\
& \times \rho_{\mathrm{W}}\left(\mathbf{R}^{\prime}, \mathbf{r}^{\prime}, \mathbf{P}, \hbar \frac{\mathbf{k}_{1}+\mathbf{k}_{2}}{2} ; t=0\right) .
\end{aligned}
$$

Reportant ce résultat dans (A10) on voit apparaitre l'intégrale :

$$
\int \mathrm{d}^{3} K \mathrm{e}^{i \mathbf{K} \cdot\left(\mathbf{R}-\mathbf{R}^{\prime}-\mathbf{P} t / M\right)}=(2 \pi)^{3} \delta\left(\mathbf{R}-\mathbf{R}^{\prime}-\mathbf{P} t / M\right)
$$

qui permet d'écrire le résultat final sous la forme :

$$
\begin{array}{r}
\rho_{\mathrm{W}}(\mathbf{R}, \mathbf{r}, \mathbf{P}, \mathbf{p} ; t)=(2 \pi)^{-3} \int \mathrm{d}^{3} \kappa \int \mathrm{d}^{3} k_{1} \int \mathrm{d}^{3} k_{2} \int \mathrm{d}^{3} r^{\prime} \mathrm{e}^{i \kappa \cdot \mathbf{r}} \mathrm{e}^{i\left(\mathbf{k}_{2}-\mathbf{k}_{1}\right) \cdot \mathbf{r}^{\prime}} \mathrm{e}^{i\left(\omega_{1}-\omega_{2}\right) t} \times \\
\times\left\langle\mathbf{k}_{+} \mid \Psi_{\mathbf{k}_{1}}^{(+)}\right\rangle\left\langle\Psi_{\mathbf{k}_{2}}^{(+)} \mid \mathbf{k}_{-}\right\rangle \rho_{\mathrm{W}}\left(\mathbf{R}-\mathbf{P} t / M, \mathbf{r}^{\prime}, \mathbf{P}, \hbar \frac{\mathbf{k}_{1}+\mathbf{k}_{2}}{2} ; t=0\right)
\end{array}
$$

d'où l'on déduit par un calcul du même type que celui du paragraphe 2 :

$$
\left[\frac{\partial}{\partial t}+\sum_{i=1}^{2} \frac{\mathbf{p}_{i}}{m} \cdot \nabla_{\mathbf{r}_{i}}\right] \rho_{\mathrm{W}}(\mathbf{R}, \mathbf{r}, \mathbf{P}, \mathbf{p} ; t)=I_{\mathrm{W}}+I_{\mathrm{W}}^{\prime}
$$

avec :

$$
\begin{aligned}
& I_{\mathrm{W}}=(2 \pi)^{-3} \int \mathrm{d}^{3} \kappa \int \mathrm{d}^{3} k_{1} \int \mathrm{d}^{3} k_{2} \int \mathrm{d}^{3} r^{\prime} \mathrm{e}^{i \kappa \cdot \mathbf{r}} \mathrm{e}^{i\left(\mathbf{k}_{2}-\mathbf{k}_{1}\right) \cdot \mathbf{r}^{\prime}} \mathrm{e}^{i\left(\omega_{1}-\omega_{2}\right) t} \times \\
& \quad \times\left\langle\mathbf{k}_{+} \mid \Psi_{\mathbf{k}_{1}}^{(+)}\right\rangle\left\langle\Psi_{\mathbf{k}_{2}}^{(+)} \mid \mathbf{k}_{-}\right\rangle \hbar\left[\frac{k_{+}^{2}-k_{-}^{2}}{2 \mu}\right] \rho_{\mathrm{W}}\left(\mathbf{R}-\mathbf{P} t / M, \mathbf{r}^{\prime}, \mathbf{P}, \hbar \frac{\mathbf{k}_{1}+\mathbf{k}_{2}}{2} ; t=0\right)
\end{aligned}
$$

et, pour $I_{\mathrm{W}}^{\prime}$ une expression semblable, où le crochet dans l'intégrale est remplacé par l'opérateur différentiel $D_{T}^{\prime}$ (déjà introduit à la fin du paragraphe 2) de dérivée totale par rapport aux variables de la fonction $\rho_{\mathrm{W}}$ qui le suit [variables de (28) pour la particule relative ; pour le centre de masse, il y a compensation entre le terme de gradient et la dérivée temporelle, à cause du terme en $-\mathbf{P} t / M$ dans le second membre de (A17)]; l'intégrale $I_{\mathrm{W}}^{\prime}$ est, pour la véritable transformée de Wigner, l'analogue de $I_{\mathrm{c}}^{\prime}$ défini en (31). A titre de comparaison, l'équivalent de (A15a) pour la transformée de Wigner libre aurait donné :

$$
\begin{aligned}
\rho_{\mathrm{W}}^{\mathrm{L}}(\mathbf{R}, \mathbf{r}, \mathbf{P}, \mathbf{p} ; t)=(2 \pi)^{-3} \int \mathrm{d}^{3} \kappa \int \mathrm{d}^{3} k_{1} \int \mathrm{d}^{3} k_{2} \int \mathrm{d}^{3} r^{\prime} \mathrm{e}^{i \kappa \cdot(\mathbf{r}-\mathbf{p} t / \mu)} \mathrm{e}^{i\left(\mathbf{k}_{2}-\mathbf{k}_{1}\right) \cdot \mathbf{r}^{\prime}} \times \\
\times\left[Y^{(+)}(\hat{\mathbf{p}} \cdot \mathbf{r}) S\left(\mathbf{k}_{+}, \mathbf{k}_{1}\right) S^{*}\left(\mathbf{k}_{-}, \mathbf{k}_{2}\right)+Y^{(-)}(\hat{\mathbf{p}} \cdot \mathbf{r}) \delta\left(\mathbf{k}_{+}-\mathbf{k}_{1}\right) \delta\left(\mathbf{k}_{-}-\mathbf{k}_{2}\right)\right] \\
\quad \times \rho_{\mathrm{W}}\left(\mathbf{R}-\mathbf{P} t / M, \mathbf{r}^{\prime}, \mathbf{P}, \hbar \frac{\mathbf{k}_{1}+\mathbf{k}_{2}}{2} ; t=0\right)
\end{aligned}
$$


et, pour l'évolution temporelle, des intégrales de collision très semblables aux intégrales $I_{\mathrm{c}}$ et $I_{\mathrm{c}}^{\prime}$ définies en (22) et (31), la seule différence étant l'insertion du temps $t$ qui correspond au temps écoulé depuis l'instant initial avant la collision, qu'il n'a pas été nécessaire d'introduire dans les calculs du paragraphe 2. De façon générale, les deux familles de formules sont assez semblables, mais présentent aussi des différences importantes. Par exemple, dans les deux cas, le centre de masse se propage de façon très simple, comme on pouvait s'y attendre physiquement, mais cette évolution simple est également applicable à $\mathbf{r}$ pour la transformée libre. Une autre différence notable est la présence dans (A15a) des coefficients :

$$
\left\langle\mathbf{k}^{\prime} \mid \Psi_{\mathbf{k}}^{(+)}\right\rangle=\delta\left(\mathbf{k}-\mathbf{k}^{\prime}\right)+\frac{1}{\hbar\left(\omega_{k}-\omega_{k^{\prime}}\right)+i \varepsilon} T\left(\mathbf{k}^{\prime}, \mathbf{k}\right)
$$

au lieu des éléments de la matrice $S$. Si les termes de degré zéro en $T$ sont les mêmes pour l'évolution des deux transformées, les termes linéaire ou quadratique sont notablement différents, et en particulier ne contiennent plus de fonction delta de l'énergie cinétique pour l'évolution de la véritable transformée de Wigner.

On peut effectuer sur (A15a) les mêmes calculs que ceux que nous avons développés dans cet article ; ils sont cependant plus lourds du fait de l'absence de fonctions delta de l'énergie cinétique, et nous ne donnerons ici qu'un aperçu des résultats. Si l'on se limite au calcul des termes d'ordre zéro en gradients spatiaux, les calculs ne sont pas très différents, et ils aboutissent d'ailleurs exactement au même résultat : on retrouve l'intégrale de collision de Boltzmann. Ainsi voyons-nous à nouveau que, tant que les collisions sont traitées comme ponctuelles, la transformée de Wigner habituelle est bien adaptée à l'écriture d'une équation cinétique. A l'ordre un en gradients, il en va cependant tout autrement. Par exemple, il apparaît dans les termes de diffusion latérale une contribution qui s'écrit :

$$
\begin{aligned}
-\frac{8 \pi^{3}}{\hbar^{2}} \int \mathrm{d}^{3} k \mathfrak{T} \frac{1}{\omega_{k}-\omega_{p}}\left[\nabla_{k}|T(\mathbf{p} / \hbar, \mathbf{k})|^{2}+|T(\mathbf{p} / \hbar, \mathbf{k})|^{2} \nabla_{\mathbf{p}}\right] \cdot \\
\cdot \nabla_{\mathrm{r}} \rho_{\mathrm{W}}\left(\mathbf{R}=\mathbf{r}_{1}-\mathbf{p} t / M, \mathbf{r}=-\hbar \mathbf{k} t / \mu, \mathbf{P}, \hbar \mathbf{k} ; t=0\right)
\end{aligned}
$$

où interviennent les éléments de matrice «off shell» de $T$ (effets des «transitions virtuelles »); le $\mathfrak{T}$ dénote une partie principale. De façon générale, la structure du second membre de l'équation est plus compliquée, ce qui physiquement correspond au fait qu'elle doit prendre en compte les transitions virtuelles et les corrélations à courte distance, alors qu'elles seront prises en compte à un stade ultérieur dans le formalisme de la transformée libre.

\section{Remarques.}

(i) Bien sûr, si l'on suppose que les particules se sont déjà éloignées de sorte qu'elles ont donc cessé d'interagir à l'instant $t$, de nouvelles simplifications se produisent; l'effet des transitions virtuelles disparaît, et de façon générale les deux types de calcul redeviennent automatiquement équivalents puisque les différences entre transformée libre et ordinaire n'existent que pendant l'interaction. Calculer l'évolution longtemps après la collision en fonction de la transformée de Wigner longtemps avant se fait aussi bien dans un formalisme que dans l'autre.

(ii) En revanche, il faut être conscient de la réelle simplification que nous avons apportée aux calculs relatifs à la véritable transformée en introduisant un instant initial 0 qui est choisi longtemps avant collision. Ce procédé nous a permis de remplacer dans (A12) les états stationnaires de diffusion par des ondes planes libres. Nous avons ainsi calculé l'évolution 
pendant la collision en fonction de l'état du système longtemps avant. Si l'on désire réellement exprimer l'évolution de la transformée de Wigner en fonction de sa valeur au même instant, deux points de vue sont possibles.

( $\alpha$ ) On peut effectivement le faire en introduisant deux relations de fermeture supplémentaires dans (A12) afin de pouvoir utiliser à nouveau les relations d'inversion (A6). Alors, le résultat final qui remplace (A15a) contient deux intégrales supplémentaires et quatre coefficients (A16) au lieu de deux :

$$
\begin{aligned}
& \frac{\partial}{\partial t} \rho_{\mathrm{W}}(\mathbf{R}, \mathbf{r}, \mathbf{P}, \mathbf{p} ; t)=(2 \pi)^{-3} \int \mathrm{d}^{3} \kappa \int \mathrm{d}^{3} k_{1} \int \mathrm{d}^{3} k_{2} \int \mathrm{d}^{3} k_{1}^{\prime} \int \mathrm{d}^{3} k_{2}^{\prime} \int \mathrm{d}^{3} r^{\prime} \times \\
& \quad \times \mathrm{e}^{i \kappa \cdot \mathbf{r}}\left[i\left(\omega_{2}-\omega_{1}\right)\right] \mathrm{e}^{i\left(\mathbf{k}_{2}^{\prime}-\mathbf{k}_{1}^{\prime}\right) \cdot \mathbf{r}^{\prime}} \\
& \quad \times\left\langle\mathbf{k}_{+} \mid \Psi_{\mathbf{k}_{1}}^{(+)}\right\rangle\left\langle\Psi_{\mathbf{k}_{1}}^{(+)} \mid \mathbf{k}_{1}^{\prime}\right\rangle\left\langle\mathbf{k}_{2}^{\prime} \mid \Psi_{\mathbf{k}_{2}}^{(+)}\right\rangle\left\langle\Psi_{\mathbf{k}_{2}}^{(+)} \mid \mathbf{k}_{-}\right\rangle \rho_{\mathrm{W}}\left(\mathbf{R}, \mathbf{r}^{\prime}, \mathbf{P}, \hbar \frac{\mathbf{k}_{1}^{\prime}+\mathbf{k}_{2}^{\prime}}{2} ; t\right) .
\end{aligned}
$$

Le calcul de la dérivée totale peut être fait comme pour (A16) et (A17), mais il est plus compliqué, en particulier parce que les fréquences de Bohr $\omega_{2}$ et $\omega_{1}$ qui sont présentes dans le terme en partie principale de (A18) ne sont plus celles qui permettent de reconstruire, après intégration par parties, le terme de dérivée spatiale qui dans $D_{\mathrm{T}}^{\prime}$ correspond à la particule relative.

( $\beta$ ) Si l'on veut éviter ces intégrales supplémentaires, on peut également remarquer que dans (A10) se trouvent directement les éléments de matrice de $\left[\Omega^{(+)}\right]^{\dagger} \rho\left[\Omega^{(+)}\right]$, qui s'expriment simplement en fonction de la transformée de Wigner libre entrante. On est ainsi conduit à un calcul mixte où l'évolution de la véritable transformée s'exprime en fonction des valeurs au même instant d'une autre transformée; le résultat est alors simplement une équation très semblable à (A15a), à ceci près que $\rho_{\mathrm{W}}$ est remplacé par $\rho_{\mathrm{WL}}^{(+)}$, calculée au même

instant $t$, et que l'exponentielle $\mathrm{e}^{i\left(\omega_{1}-\omega_{2}\right) t}$ disparaît. Ces remarques illustrent bien la simplification apportée par la transformée libre pour un calcul exact de l'évolution pendant l'interaction des deux particules.

\section{Bibliographie}

[1] HuANG K., Statistical mechanics (Wiley) 1963, Chapitre 5.

[2] Beth E. and Uhlenbeck G. E., Physica 4 (1937) 915.

[3] Référence [1], paragraphe 14.3.

[4] Voir par exemple la discussion donnée dans Cohen-Tannoudji, Diu et Laloë, Mécanique quantique, Hermann (1973), complément $J_{I}$ et chapitre VIII, paragraphe B.

[5] Hoffmann D., Muller J. and Curtiss C., J. Chem. Phys. 43 (1965) 2878.

[6] Snider R. F., J. Chem. Phys. 32 (1960) 1051.

[7] Hess S., Z. Naturforsch. 22a (1967) 1871.

[8] Baerwinkel K. and Grossmann S., Z. Phys. 198 (1967) 277.

[9] Thomas M. W. and Snider R. F., J. Chem. Phys. 2 (1970) 61.

[10] Meyerovich A., proceedings of the Spin Polarized Quantum Systems conference in Torino, Ed. S. Stringari (World Scientific) (1988) ; Phys. Rev. B 39 (1989) 9318.

[11] Wigner E. P., Phys. Rev. 40 (1932) 749.

[12] Imre K., Ozizmir E., Rosenbaum M. and Zweifel P. F., Journ. Math. Phys. 8 (1967) 1097.

[13] Hillery M., O’Connell R. F., Scully M. O. and Wigner E. P., Phys. Rep. 106 (1984) 121-167.

[14] Balazs N. L. and Jennings B. K., Physics Rep. 104 (1984) 347-391.

[15] Groenewold H. J., Physica 12 (1946) 405. 